\title{
Agnostic Learning in Permutation-Invariant Domains
}

KARL WIMMER, Duquesne University

We generalize algorithms from computational learning theory that are successful under the uniform distribution on the Boolean hypercube $\{0,1\}^{n}$ to algorithms successful on permutation-invariant distributions, distributions that stay invariant constant on permutating the coordinates in the instances. While the tools in our generalization mimic those used for the Boolean hypercube, the fact that permutation-invariant distributions are not product distributions presents a significant obstacle.

We prove analogous results for permutation-invariant distributions; more generally, we work in the domain of the symmetric group. We define noise sensitivity in this setting and show that noise sensitivity has a nice combinatorial interpretation in terms of Young tableaux. The main technical innovations involve techniques from the representation theory of the symmetric group, especially the combinatorics of Young tableaux. We show that low noise sensitivity implies concentration on "simple" components of the Fourier spectrum and that this fact will allow us to agnostically learn halfspaces under permutation-invariant distributions to constant accuracy in roughly the same time as in the uniform distribution over the Boolean hypercube case.

Categories and Subject Descriptors: F.2.m [Analysis of Algorithms and Problem Complexity]: Miscellaneous

General Terms: Algorithms, Theory

Additional Key Words and Phrases: Learning theory, Fourier analysis, representation theory of the symmetric group

ACM Reference Format:

Karl Wimmer. 2016. Agnostic learning in permutation-invariant domains. ACM Trans. Algorithms 12, 4, Article 46 (August 2016), 22 pages.

DOI: http://dx.doi.org/10.1145/2963169

\section{INTRODUCTION}

In this article, we:

- Generalize the Low-Degree algorithm (and the agnostic learning algorithm of Kalai et al. [2008]) to the symmetric group, taking special care to account for the fact that the Fourier spectrum consists of matrices.

-Generalize the concept of noise sensitivity for a function $f: S_{n} \rightarrow \mathbb{R}$ and give an expression for noise sensitivity in terms of the Fourier spectrum of such functions.

-Prove that the noise sensitivity of generalized linear threshold functions $f: S_{n} \rightarrow$

$\mathbb{R}$ have bounded noise sensitivity and that this theorem will allow us to use the algorithm of Kalai et al. [2008] to agnostically learn these functions.

Our primary motivation is the class of binary classification problems over the instance space $\mathcal{X}=X^{n}$ for some set $X=\left\{X_{1}, X_{2}, \ldots, X_{t}\right\}$ with $t=\operatorname{poly}(n)$. Consider the following algorithm for learning in such a scenario:

A preliminary version of this work appeared in FOCS 2010 titled "Agnostically learning under permutation invariant distributions."

Author's address: K. Wimmer, 440 College Hall, 600 Forbes Avenue, Pittsburgh, PA 15282.

Permission to make digital or hard copies of part or all of this work for personal or classroom use is granted without fee provided that copies are not made or distributed for profit or commercial advantage and that copies show this notice on the first page or initial screen of a display along with the full citation. Copyrights for components of this work owned by others than ACM must be honored. Abstracting with credit is permitted. To copy otherwise, to republish, to post on servers, to redistribute to lists, or to use any component of this work in other works requires prior specific permission and/or a fee. Permissions may be requested from Publications Dept., ACM, Inc., 2 Penn Plaza, Suite 701, New York, NY 10121-0701 USA, fax +1 (212) 869-0481, or permissions@acm.org.

(c) 2016 ACM 1549-6325/2016/08-ART46 $\$ 15.00$

DOI: http://dx.doi.org/10.1145/2963169 
Given $m$ examples of training data $\left(\vec{x}_{1}, y_{1}\right), \ldots,\left(\vec{x}_{m}, y_{m}\right) \in \mathcal{X} \times\{-1,1\}$,

(1) Expand each instance $\vec{x}_{i}$ into a vector from $\{0,1\}^{n|X|}$, where each entry is the indicator of $\vec{x}_{i}=X_{j}$.

(2) Consider new "features" that are products of up to $d$ of these $0 / 1$ attributes.

(3) Find the linear function $W$ in the feature space that best fits the training labels under some loss measure $\ell$, such as squared loss, hinge loss, or $L_{1}$ loss.

(4) Output the hypothesis $\operatorname{sgn}(W-\theta)$, where $\theta \in[-1,1]$ is chosen to minimize the hypothesis' training error.

We note that the above algorithm, which we will refer to as polynomial regression (as in Blais et al. [2010]), is a version of the wildly popular Support Vector Machines (SVM) algorithm. It is known that the above algorithm runs in $\operatorname{poly}\left(m, n^{d}\right)$ with $m$ training examples. If $m=\Omega\left(n^{d} / \epsilon\right)$, then the SVM algorithm is guaranteed (with high probability) to generalize to unseen data in the case that $\mathcal{X}$ is a product distribution [Blais et al. 2010]. In fact, in Blais et al. [2010], the domain $\mathcal{X}$ is allowed to be the product of different sets, which can be of any finite cardinality (and even uncountably infinite under distributional assumptions). Further, to achieve any provable guarantee that the hypothesis generalizes, $\Theta\left(n^{d} / \epsilon\right)$ examples are necessary.

In the literature, much effort has been put into the case where the attributes are mutually independent. In this case, the data are drawn i.i.d. from a product distribution over $\mathcal{X}$. The uniform distribution over $\{0,1\}^{n}$ has received much attention in a number of different scenarios; some of these results extend to product distributions. This was the explicit motivation of Blais et al. [2010]. In Kalai et al. [2009], the authors show that learning of some natural concepts is possible under a randomly chosen product distribution with high probability. In practice, the assumption that the attributes are all mutually independent is unrealistic.

The assumption we make in this work is that the distribution is permutation invariant. By this, we mean that $\boldsymbol{P r}_{\boldsymbol{X}}\left[\boldsymbol{X}=\left(\boldsymbol{x}_{1}, \boldsymbol{x}_{2}, \ldots, \boldsymbol{x}_{n}\right)\right]=\operatorname{Pr}_{\boldsymbol{X}}\left[\boldsymbol{X}=\left(\boldsymbol{x}_{\sigma(1)}, \boldsymbol{x}_{\sigma(2)}, \ldots, \boldsymbol{x}_{\sigma(n)}\right)\right]$ for any permutation $\sigma$. A permutation-invariant distribution need not even be pairwise independent, although product distributions with equal biases for each coordinate are permutation invariant. Considering this type of distribution has already proven helpful in other work. In the context of learning monotone functions over the uniform distribution on $\{0,1\}^{n}$, O'Donnell and Wimmer [2009] show that product distributions are too spread out for a natural approach to work. Converting to permutation-invariant distributions of the previously mentioned form allows for stronger analysis. The approach in O'Donnell and Wimmer [2009] is to learn monotone functions over one level of the Boolean cube at a time, focusing on each collection of examples with a fixed number of 1 's.

To prove our results, we use tools from representation theory over the symmetric group. We define a notion of noise sensitivity for the symmetric group and achieve similar learning results to those over the uniform distribution. The results and connections between computational learning theory and representation theory are interesting, and the combinatorial results we show relating to the symmetric group might be of independent interest. We were unable to find a reference to our notion of noise sensitivity in the literature; for example, it differs from the content found in Diaconis [1988].

Definition 1.1. For a function $f: S_{n} \rightarrow\{-1,1\}$ and a parameter $0<\delta<1$, we define the noise sensitivity of $f$ at $\delta$, denoted $\mathbb{N S}_{\delta}(f)$, in the following way: Let $\alpha \sim$ $\operatorname{Binomial}(n, \delta)$, and choose a uniformly random subset $\boldsymbol{S}$ of $[n]$ of cardinality $\boldsymbol{\alpha}$. Let $\boldsymbol{\psi}$ be a uniformly random permutation in $S_{n}$ such that every element of $[n] \backslash \boldsymbol{S}$ is a fixed 
point of $\psi$. Then

$$
\mathbb{N} \mathbb{S}_{\delta}(f)=\underset{\sigma, \psi}{\operatorname{Pr}}[f(\sigma) \neq f(\psi \sigma)],
$$

where $\sigma$ is chosen uniformly at random from $S_{n}$.

We have used $[n]$ to denote the set $\{1,2, \ldots, n\}$.

\subsection{The Learning Framework}

Our goal is binary classification learning in the "agnostic" model introduced in Kearns et al. [1994]. In this model, there is an unknown target function $t: \mathcal{X} \rightarrow\{-1,1\}$ that we are trying to recover. Our access to $t$ is limited; we receive labeled examples of the form $\langle x, t(x)\rangle$, where the marginal distribution on the first coordinate is some known distribution $\mathcal{D}$ on the set $\mathcal{X}$. Further, the examples are generated independently. The algorithm's task is to output a hypothesis $h: \mathcal{X} \rightarrow\{-1,1\}$ with minimal classification error with respect to $t$; that is, we wish to choose $h$ minimizing $\operatorname{err}_{t}(h)=\operatorname{Pr}_{\boldsymbol{x} \sim \mathcal{D}}[h(\boldsymbol{x}) \neq$ $t(\boldsymbol{x})]$. We compare the error of our hypothesis to the minimum error achievable using a function from some fixed class $\mathcal{C}$ of functions from $\mathcal{X}$ to $\{-1,1\}$. We say that we can "agnostically learn with respect to $\mathcal{C}$ under the distribution $\mathcal{D}$ " if, for any target function $t$, any $\epsilon>0$, given a bounded number of labeled examples, our algorithm returns a hypothesis satisfying

$$
\mathbf{E}\left[\operatorname{err}_{t}(h)\right] \leq \inf _{f \in C} \operatorname{err}_{t}(f)+\epsilon,
$$

where the expectation is over the randomness of our algorithm. Our goal is to minimize the time and examples required for agnostically learning a class $\mathcal{C}$.

In light of strong computational hardness results for such problems when the distribution is arbitrary, much work has gone into the case where the distribution $\mathcal{D}$ is a product distribution; that is, a distribution where all of the coordinates are mutually independent. The advantage of such a distribution is the ease with which an orthogonal decomposition can be constructed and analyzed.

\section{ALGORITHMS FOR LEARNING}

We recount some algorithms for learning. First, we make a definition:

Definition 2.1. For a function $f:\{0,1\}^{n} \rightarrow\{-1,1\}$, we say that $f$ is $\epsilon$-concentrated to degree $d$ with respect to $\mathcal{D}$ if there exists a polynomial $p$ of degree at most $d$ such that $\mathbf{E}_{\boldsymbol{x} \sim \mathcal{D}}\left[(f(\boldsymbol{x})-p(\boldsymbol{x}))^{2}\right] \leq \epsilon$. We say that a class of functions $\mathcal{C}$ is $\epsilon$-concentrated to degree $d$ if every $f \in \mathcal{C}$ is $\epsilon$-concentrated to degree $d$.

The concept of $\epsilon$-concentration has proved fruitful in computational learning theory. The Low-Degree Algorithm of Linial et al. [1993] was the first result to use $\epsilon$-concentration. In their article, the authors considered learning under the uniform distribution on $\{0,1\}^{n}$. They show that if the target function $t$ is computable by a size $s$, depth $c$ circuit, then $t$ is $\epsilon$-concentrated to degree $O(\log (s / \epsilon))^{c}$.

While our results can be applied to learning circuits, our primary focus here is the class of linear threshold functions.

Definition 2.2. We say that a function $f:\{0,1\}^{n} \rightarrow\{-1,1\}$ is a linear threshold function if there exists weights $w_{1}, w_{2}, \ldots, w_{n}$ and a threshold $\theta$ such that $f(x)=$ $\operatorname{sgn}\left(\sum_{i=1}^{n} w_{i} x_{i}-\theta\right)$ for all $x \in\{0,1\}^{n}$.

Klivans et al. [2004] develop the "noise sensitivity method" along these lines. They show $\epsilon$-concentration results for functions with bounded noise sensitivity. Specifically, they show that any function $f:\{0,1\}^{n} \rightarrow\{-1,1\}$ that can be written as a function 
of $k$ linear threshold functions is $\epsilon$-concentrated to degree $O\left(k^{2} / \epsilon^{2}\right)$ under the uniform distribution on $\{0,1\}^{n}$.

At the time of writing of Klivans et al. [2004], it was not known if any $\epsilon$-concentration results had implications in the agnostic learning framework. For applications in computational learning theory, results were possible with the extra assumption that the target function $t$ was in $\mathcal{C}$; specifically, $\mathcal{C}$ is the class of functions computable by some size $s$, depth $c$ circuit (to apply Linial et al. [1993]) or computable by function of $k$ linear threshold functions (to apply Klivans et al. [2004]).

The work in Kalai et al. [2008] gives a significant step forward in this line of work. In their article, they consider the agnostic problem, where we do not assume $t \in \mathcal{C}$. In that article, the authors show that $L_{2}$-approximability bounds can be used to imply $L_{1^{-}}$ approximability bounds, which can be used to achieve results in the agnostic setting. Under the uniform distribution on $\{0,1\}^{n}$, they show that $\epsilon^{2}$-concentration up to degree $d$ implies agnostic learning with accuracy loss $\epsilon$. Their algorithm differs slightly from the Low-Degree algorithm; their algorithm solves a linear program for a degree- $d$ polynomial $p(x)$ that minimizes absolute $L_{1}$ error. They also note that this is a version of the SVM algorithm.

A further step in this direction is given in Blais et al. [2010]. Blais, O'Donnell, and Wimmer show that nearly all results about $\epsilon$-concentration with respect to the uniform distribution on $\{0,1\}^{n}$ can be applied to arbitrary product distributions; distributions where the coordinates are mutually independent, but each coordinate has an arbitrary distribution. The main tools therein are an extension of the noise sensitivity method of Klivans et al. [2004] to product distributions and an application of the algorithm of Kalai et al. [2008]. In this article, we use both of these techniques, suitably adjusted for functions over the symmetric group. We also note the work of Kane et al. [2013], which show results similar to $\epsilon$-concentration. Most of their effort applies to functions over continuous spaces rather than discrete, although some of their results apply to learning halfspaces over $k$-wise independent distributions over the hypercube where $k=\Theta\left(1 / \epsilon^{4}\right)$. We also note the work of Feldman and Kothari [2015] in agnostically learning disjunctions (which are a special case of linear threshold functions) over permutation-invariant distributions. For this restricted class of linear threshold functions, they achieve much better running time without appealing to $\epsilon$-concentration techniques.

The main technique we will use comes from representation theory of the symmetric group. We recall the work of Boneh [1995], which used representation theory of groups of size $2^{n}$ to establish learning results for the uniform distribution on the hypercube. The symmetric group is not mentioned in Boneh's article. We note that learning results of a more applied nature are known for the symmetric group, particularly in the realm of multi-object tracking [Kondor and Borgwardt 2008; Kondor et al. 2007; Huang et al. 2009]. All of these results make heavy use of representation theory; this document combines the techniques used in these works with the tools and ideas from the field of computational learning theory. Further, Shivaswamy and Jebara [2006] introduce a similar idea that they call permutation-invariant SVMs, where the classifier is forced to be invariant under permutations. We note that our main assumption is that the distribution is invariant under permutations, but the classifier need not be.

\section{OVERVIEW OF RESULTS}

The main result of this article is the following:

THEOREM 3.1. Let $\mathcal{C}$ be the class of functions of $k$ linear threshold functions over $\{0,1\}^{n}$, and let $\mathcal{D}$ be any permutation-invariant distribution over $\{0,1\}^{n}$. There is an algorithm 
that agnostically learns with respect to $\mathcal{C}$ under the distribution $\mathcal{D}$, using $n^{O\left(k^{2} / \epsilon^{4}\right)}$ time and examples.

Our main theorem is actually a corollary of a stronger result, which applies to the symmetric group. We first need the definition of linear threshold function for this domain.

Definition 3.2. We say that a function $f: S_{n} \rightarrow\{-1,1\}$ is a linear threshold function if there exist weights $w_{i j}$ for $1 \leq i, j \leq n$ and a threshold $\theta$ such that $f(\sigma)=\operatorname{sgn}\left(\sum_{i, j} w_{i j} \mathbf{1}[\sigma(i)=j]-\theta\right)$ for all $\sigma \in S_{n}$.

Theorem 3.3. Let $\mathcal{C}$ be the class of functions of $k$ linear threshold functions over $S_{n}$. There is an algorithm that agnostically learns $\mathcal{C}$ under the uniform distribution on $S_{n}$, using $n^{O\left(k^{2} / \epsilon^{4}\right)}$ time and examples.

We will prove our main theorem as a corollary of Theorem 3.3. We also prove the following generalization.

Definition 3.4. We say that a function $f:\{1,2, \ldots, B\}^{n} \rightarrow\{-1,1\}$ is a linear threshold function if there exist weights $w_{i j}$ for $1 \leq i \leq n, 1 \leq j \leq B$ and a threshold $\theta$ such that $f(x)=\operatorname{sgn}\left(\sum_{i, j} w_{i j} \mathbf{1}\left[x_{i}=j\right]-\theta\right)$ for all $x \in\{1,2, \ldots, B\}^{n}$.

THEOREM 3.5. Let $\mathcal{C}$ be the class of functions of $k$ linear threshold functions over $\mathcal{X}=\{1,2, \ldots, B\}^{n}$, and let $\mathcal{D}$ be any permutation-invariant distribution over $\mathcal{X}$. There is an algorithm that agnostically learns with respect to $\mathcal{C}$ under the distribution $\mathcal{D}$, using $n^{O\left(k^{2} / \epsilon^{4}+B\right)}$ time and examples.

We note that in a permutation-invariant distribution, the attributes need not even be pairwise independent. The class of permutation-invariant distributions includes some product distributions as a special case. In some sense, permutation-invariant distributions are a generalization of $p$-biased distributions.

To prove the above theorems, we prove the following generalization of Peres' Theorem [Peres 2004]:

Theorem 3.6. Let $f: S_{n} \rightarrow\{-1,1\}$ be a linear threshold function. Then $\mathbb{N S}_{\delta}(f) \leq$ $O(\sqrt{\delta})$.

We note that the bound $O(\sqrt{\delta})$ is the same bound for product distribution versions of this theorem. The uniform distribution case is discussed in Peres [2004], and the arbitrary product distribution case is proved in Blais et al. [2010]. In all of these results, the hidden constant is less than 2.

Most of our efforts will be put into learning functions where the domain is $S_{n}$; we reduce to other distributions by identifying letters of the permutation. For example, if we can learn under the uniform distribution over permutations of $(1,2,3, \ldots, n)$, it is intuitively plausible that we can learn under the uniform distribution on permutations of $1^{n / 2} 0^{n / 2}$ (here we mean the vector of $n / 21$ 's followed by $n / 20$ 's) by identifying $\{1,2,3, \ldots, n / 2\}$ as indistinguishable as well as $\{n / 2+1, n / 2+2, \ldots, n\}$. This is indeed the case for the classes of functions we consider. Using the same technique as seen in Blais et al. [2010] for learning under mixtures of distributions, we prove the main theorem.

\section{PRELIMINARIES}

\subsection{Representation Theory}

We begin with representation theory of general groups. 
Definition 4.1. We say that a representation $\rho$ of a group $G$ is a mapping $\rho: G \rightarrow$ $\mathbb{R}^{d_{\rho} \times d_{\rho}}$, which preserves the algebraic structure of $G$; that is, for $\sigma_{1}, \sigma_{2} \in G$ we have $\rho\left(\sigma_{1} \sigma_{2}\right)=\rho\left(\sigma_{1}\right) \cdot \rho\left(\sigma_{2}\right)$. The matrices in the codomain of $\rho$ are called the representation matrices, and $d_{\rho}$ is the degree of the representation.

Definition 4.2. The character $\chi_{\rho}$ of a representation $\rho$ is a mapping $\chi_{\rho}: G \rightarrow \mathbb{R}$ such that $\chi_{\rho}(\sigma)=\operatorname{tr} \rho(\sigma)$.

We will be concerned with representations that are irreducible. These are representations that cannot be decomposed into simpler representations. We say that two representations $\rho_{1}$ and $\rho_{2}$ are equivalent if there exists a nonsingular matrix $C$ such that $\rho_{2}(\sigma)=C^{-1} \rho_{1}(\sigma) C$ for all $\sigma \in G$. A representation $\rho$ is irreducible if we cannot write

$$
\rho=C^{-1}\left[\begin{array}{cc}
\rho_{1} & 0 \\
0 & \rho_{2}
\end{array}\right] C
$$

for some nonsingular matrix $C$ and two lower degree representations $\rho_{1}$ and $\rho_{2}$. A set of all possible irreducible representations such that no two are equivalent is called a complete set of irreducible representations of $G$. We will not be concerned with any specific complete set of irreducible representations, but we mention that there are certain canonical choices for certain groups. It is well known that if $G$ is abelian, then all the irreducible representations have degree 1. For a finite group, the functions given in the matrix entries of a complete set of irreducible representations of $G$ form an orthogonal (but not necessarily orthonormal) basis for $L^{2}(G)$; this is a consequence of the Peter-Weyl Theorem (which applies more generally to compact topological groups). We will only consider finite groups here.

Definition 4.3. Let $f: G \rightarrow \mathbb{R}$ be any function on $G$, and let $\rho$ be any representation of $G$. The Fourier coefficient of $f$ at the representation $\rho$ is given by the matrix ${ }^{1}$

$$
\widehat{f}_{\rho}=\frac{1}{|G|} \sum_{\sigma \in G} f(\sigma) \rho(\sigma) .
$$

The collection of the matrices $\widehat{f}_{\rho}$ at irreducible representations of $G$ is called the Fourier transform of $f$.

We can reconstruct $f$ from its Fourier transform via the Fourier Inversion formula:

$$
f(\sigma)=\sum_{\rho} d_{\rho} \operatorname{tr}\left(\widehat{f}_{\rho}^{T} \rho(\sigma)\right) .
$$

The tr expression can be thought of as a dot product between two length- $d_{\rho}^{2}$ vector versions of $\widehat{f}_{\rho}$ and $\rho$, arranging each matrix into a vector by taking the elements first top to bottom and then left to right as vectors.

\subsection{Boolean Hypercube}

In the Boolean hypercube case, we consider functions of the form $f: \mathbb{Z}_{2}^{n} \rightarrow\{-1,1\}$. We think of the group $\mathbb{Z}_{2}^{n}$ as generated by the $n$ standard basis vectors of the form $e^{i}=(0, \ldots, 0,1,0, \ldots, 0)$, where $\left(e^{i}\right)_{i}=1$. Since $\mathbb{Z}_{2}^{n}$ is abelian, all the representations have degree 1 , and it is common to identify representations and characters; we use the symbol $\chi$. There are $2^{n}$ inequivalent irreducible representations, which are in bijection

\footnotetext{
${ }^{1}$ This definition is slightly nonstandard; the division by $|G|$ is usually omitted. We use this definition to retain similarity to the literature on the Boolean hypercube.
} 
with $\mathbb{Z}_{2}^{n}$ in a natural way. For $\alpha \in\{0,1\}^{n}$, we define $\chi_{\alpha}: \mathbb{Z}_{2}^{n} \rightarrow\{-1,1\}$ such that $\chi_{\alpha}(x)=(-1)^{\sum_{i=1}^{n} \alpha_{i} x_{i}}$ (treating elements of $\mathbb{Z}_{2}$ as real numbers 0 and 1 in the natural way). We consider the vector space $L^{2}\left(\mathbb{Z}_{2}^{n}\right)$, and we equip this vector space with the inner product defined by $\langle f, g\rangle=\mathbf{E}_{x}[f(\boldsymbol{x}) g(\boldsymbol{x})]$, where $\boldsymbol{x}$ has the uniform distribution. The functions $\left\{\chi_{\alpha}\right\}$ form an orthonormal basis for this vector space, and it follows that every function $f: \mathbb{Z}_{2}^{n} \rightarrow \mathbb{R}$ can be uniquely written $f=\sum_{\alpha \in\{0,1\}^{n}} \hat{f}(\alpha) \chi_{\alpha}$ for some constants $\hat{f}(\alpha)$. These constants are called the Fourier coefficients of $f$, and together make up the Fourier spectrum of $f$.

We have Parseval's identity, which implies that $\sum_{\alpha} \hat{f}(\alpha)^{2}=1$ for a function $f$ : $\mathbb{Z}_{2}^{n} \rightarrow\{-1,1\}$. This is a special case of Plancherel's identity, which states that $\langle f, g\rangle=$ $\mathbf{E}_{\boldsymbol{x}}[f(\boldsymbol{x}) g(\boldsymbol{x})]=\sum_{\alpha} f(\alpha) g(\alpha)$ for $f, g: \mathbb{Z}_{2}^{n} \rightarrow \mathbb{R} ;$ Parseval follows by taking $g=f$ and noticing that $f^{2} \equiv 1$.

For a subset $S$ of $\{0,1\}^{n}$, we say that a function $f: \mathbb{Z}_{2}^{n} \rightarrow\{-1,1\}$ is $\epsilon$-concentrated on $S$ if $\sum_{\alpha \in S} \hat{f}(\alpha)^{2} \geq 1-\epsilon$. Let $f_{S}=\sum_{\alpha \in S} \hat{f}(\alpha) \chi_{\alpha}$. Then, for $f: \mathbb{Z}_{2}^{n} \rightarrow \mathbb{R}$, we have $\mathbf{E}_{\boldsymbol{x}}\left[\left(f(\boldsymbol{x})-f_{S}(\boldsymbol{x})\right)^{2}\right] \leq \epsilon$. It is well known that if $f: \mathbb{Z}_{2}^{n} \rightarrow\{-1,1\}$ is $\epsilon$-concentrated on $S$, then $\mathbf{P r}_{x}\left[f(\boldsymbol{x}) \neq \operatorname{sgn}\left(f_{S}(\boldsymbol{x})\right)\right] \leq \epsilon$.

Let $P_{d}=\left\{x \in\{0,1\}^{n}:|x| \leq d\right\}$. The span of the functions $\chi_{\alpha}$ where $\alpha \in P_{d}$ is the space of all degree- $d$ polynomials over $\mathbb{Z}_{2}^{n}$. In the case that $f$ is $\epsilon$-concentrated on $P_{d}$, we say that $f$ is $\epsilon$-concentrated to degree $d$, and we recover Definition 2.1.

For $x \in \mathbb{Z}_{2}^{n}$, we write $x^{(i)}$ to denote $x$ with the $i$ th coordinate flipped. We define the influence of a coordinate $i$ as $\operatorname{Inf}_{i}(f)=\mathbf{P r}_{x}\left[f(\boldsymbol{x}) \neq f\left(\boldsymbol{x}^{(i)}\right)\right]$, and the total influence as $\operatorname{Inf}(f)=\sum_{i=1}^{n} \operatorname{Inf}_{i}(f)$. For a set $S$, let $\boldsymbol{x}^{S}$ denote $\boldsymbol{x}$ with the coordinates of $S$ rerandomized. Then, for a parameter $0<\delta<1$, we define the noise sensitivity of $f$ at $\delta$ to be $\mathbb{N S}_{\delta}(f)=\mathbf{P r}_{\boldsymbol{x}, \boldsymbol{S}}\left[f(\boldsymbol{x}) \neq f\left(\boldsymbol{x}^{\boldsymbol{S}}\right)\right]$, where $\boldsymbol{S}$ is a uniformly randomly chosen subset of $[n]$ with cardinality $\operatorname{Binomial}(\delta, n)$. Equivalently, we can draw $\boldsymbol{x}^{\boldsymbol{S}}$ by independently rerandomizing each coordinate of $\boldsymbol{x}$ with probability $\delta$; it is easy to see that $\boldsymbol{S}$ is simply the set of coordinates rereandomized.

These quantities have nice interpretations in terms of the Fourier coefficients of $f$ :

$$
\operatorname{Inf}_{i}(f)=\sum_{\alpha: \alpha_{i}=1} \hat{f}(\alpha)^{2} \quad \operatorname{Inf}(f)=\sum_{\alpha}|\alpha| \hat{f}(\alpha)^{2} \quad \mathbb{N S}_{\delta}(f)=\sum_{\alpha}\left(\frac{1}{2}-\frac{1}{2}(1-\delta)^{|\alpha|}\right) \hat{f}(\alpha)^{2} .
$$

In the latter two expressions above, the coefficients of $\hat{f}(\alpha)^{2}$ increase as $|\alpha|$ increases, and it follows that if $\operatorname{Inf}(f)$ or $\mathbb{N S}_{\delta}(f)$ is small enough, then $f$ is $\epsilon$-concentrated to degree $d$ for sufficiently large $d$. As an example, in Peres [2004], it is proved that if $f:\{0,1\}^{n} \rightarrow\{-1,1\}$ is a linear threshold function, then $\mathbb{N S}_{\delta}(f) \leq O(\sqrt{\delta})$. It follows from Klivans et al. [2004] that any function $f:\{0,1\}^{n} \rightarrow\{-1,1\}$ with this noise sensitivity bound is $\epsilon$-concentrated on $P_{d}$ where $d=O\left(1 / \epsilon^{2}\right)$.

\subsection{The Symmetric Group and Young Tableaux}

In this section, we consider $L^{2}\left(S_{n}\right)$, the class of functions $f: S_{n} \rightarrow \mathbb{R}$, as a vector space of dimension $n$ ! under standard addition and scalar multiplication operations. We equip this space with the inner product $\langle f, g\rangle=\mathbf{E}_{\sigma}[f(\boldsymbol{\sigma}) g(\boldsymbol{\sigma})]$.

Since the symmetric group is not abelian, Fourier analysis becomes much more involved. We will be very interested in the irreducible representations of $S_{n}$. We define a partition $\lambda$ of $n$ to be a nonincreasing sequence of integers $\left(\lambda_{1}, \lambda_{2}, \ldots, \lambda_{m}\right)$, where $\sum_{i} \lambda_{i}=n$ and each $\lambda_{i}>0$. We write this as $\lambda \vdash n$. The following is well known:

THEOREM 4.4. The irreducible representations of $S_{n}$ can be indexed by partitions of $n$.

It is common to use Ferrer's diagrams to visualize a partition of $n$. Ferrer's diagrams represent each component of each partition as the number of squares in the 
corresponding row. A standard Young tableau is a Ferrer's diagram with the numbers $1,2, \ldots, n$ each occurring in a different cell, such that the numbers in the cells are increasing downwards and to the right. (We refer the reader to Fulton [1997] for a thorough treatment of Young tableaux.) We define the dominance order on partitions of $n$ in the following way: We write $\lambda \unrhd \mu$ if $\sum_{i=1}^{k} \lambda_{i} \geq \sum_{i=1}^{k} \mu_{i}$ for all $i$, where we pad the partitions with extra zeroes where necessary.

Another combinatorial concept that will be important for us is the concept of skew diagrams. Given partitions $\lambda \vdash n$ and $\mu \vdash k$ with $\lambda_{1} \geq \mu_{1}$, a skew diagram of shape $\lambda / \mu$ is the diagram formed by the set-theoretic difference of $\lambda$ and $\mu$. A skew-standard Young tableau is a skew diagram filled with the numbers $1,2, \ldots, n-k$, each number occurring in exactly one cell, such that the numbers in the cells are increasing downwards and to the right. We will only be concerned with skew tableau where $\mu=(k)$.

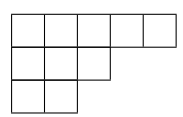

A standard diagram of shape $(5,3,2)$

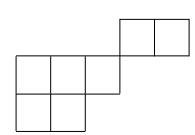

A skew diagram of shape $(5,3,2) /(3)$.

We say that the degree of a Ferrer's diagram of shape $\lambda$ is the number of standard Young tableaux of shape $\lambda$, and we denote the degree $\operatorname{dim} \lambda$ or $d_{\lambda}$. We will also refer to this as the degree of the partition $\lambda$. Similarly, we define $\operatorname{dim} \lambda / \mu$ as the number of skew-standard Young tableaux of shape $\lambda / \mu$, taking $\operatorname{dim} \lambda / \mu=0$ if $\lambda_{1}<\mu_{1}$. A variety of expressions for these dimensions are known.

Because of the equivalence of irreducible representations and partitions, we will frequently identify $\rho_{\lambda}$ and $\lambda$, where $\rho_{\lambda}$ is an irreducible representation corresponding to the class of representations indexed by the partition of $\lambda$. We will shorten $\rho_{\lambda}$ to $\rho$ when the correspondence is clear from context. The fact that we called the degree of a Ferrer's diagram $d_{\rho}$ is suggestive of the following theorem:

THEOREM 4.5. The degree of any irreducible representation is equal to the degree of the Ferrer's diagram of the corresponding partition. That is, $d_{\lambda}=d_{\rho_{\lambda}}$, which we will write as $d_{\rho}$ when $\lambda$ is clear from the context. Further, we can write $\widehat{f}_{\lambda}$ in place of $\widehat{f}_{\rho}$ or $\widehat{f}_{\rho_{\lambda}}$.

\subsection{Polynomials and $\boldsymbol{\epsilon}$-Concentration}

We define a simple function of degree $d$ to be a function $f: S_{n} \rightarrow \mathbb{R}$ such that there exists $d$ coordinates $1 \leq i_{1}<i_{2}<\ldots<i_{d} \leq n$ such that $f(\sigma)$ is completely determined by $\sigma\left(i_{1}\right), \sigma\left(i_{2}\right), \ldots, \sigma\left(i_{d}\right)$ for all $\sigma$. A function of degree $d$ is a function expressible as a linear combination of simple functions of degree $d$. Let $P_{d}^{\prime}=\left\{\lambda \vdash n: \lambda_{1} \geq n-d\right\}$. Then the span of the set of functions $\left\{\left(\rho_{\lambda}\right)_{i j}: \lambda \in P_{d}^{\prime}, 1 \leq i, j \leq d_{\lambda}\right\}$ is exactly the vector space of functions of degree $d$. We remark that a characterization of Boolean functions that are functions of degree $d$ over $S_{n}$ is given in Ellis et al. [2011].

We have Parseval's identity, which says that for $f: S_{n} \rightarrow\{-1,1\}$, we have $\sum_{\lambda \vdash n} d_{\lambda}\left\|\hat{f}_{\lambda}\right\|^{2}=1$. All matrix norms are the Frobenius norm: $\|A\|=$ $\sqrt{\operatorname{tr}\left(A^{T} A\right)}=\sqrt{\sum_{i, j} A_{i j}^{2}}$. Parseval's identity follows from Plancherel's identity: $\langle f, g\rangle=$ $\sum_{\lambda \vdash n} d_{\lambda} \operatorname{tr}\left(\hat{f}_{\lambda}^{T} \hat{g}\right)$.

We can now give the proper generalization of the Fourier interpretation of $\epsilon$ concentration. Let $S$ be a set of partitions of $n$. We say that $f: S_{n} \rightarrow\{-1,1\}$ is $\epsilon$-concentrated on $S$ if $\sum_{\lambda \in S} d_{\lambda}\left\|\hat{f}_{\lambda}\right\|^{2} \geq 1-\epsilon$. Applying Parseval's identity, $f$ is $\epsilon$ concentrated on $S$ if $\sum_{\lambda \notin S} d_{\lambda}\left\|\hat{f}_{\lambda}\right\|^{2} \leq \epsilon$. If $f$ is $\epsilon$-concentrated on $P_{d}^{\prime}$, then $f$ is $\epsilon$ concentrated to degree $d$ as in Definition 2.1. As in previous work in the literature, 
we will show that functions $f: S_{n} \rightarrow\{-1,1\}$ with low noise sensitivity achieve $\epsilon$ concentration to degree $d(\epsilon)$, where $d(\epsilon)=1 / \epsilon^{2}$.

Applying Theorem 5 of Kalai et al. [2008], we see that if $\mathcal{C}$ is a set of functions over $S_{n}$, all of which are $\epsilon$-concentrated to degree $d(\epsilon)$, then we can agnostically learn with respect to $\mathcal{C}$ under the uniform distribution on $S_{n}$ in time $n^{O\left(d\left(\epsilon^{2}\right)\right)}$. The algorithm is $L_{1}$ polynomial regression to degree $d\left(\epsilon^{2}\right)$. The basis functions can be taken to be indicator functions,

$$
\prod_{k=1}^{m} \mathbf{1}\left[\sigma\left(i_{k}\right)=j_{k}\right],
$$

where $i_{1}, i_{2}, \ldots, i_{k} \in[n], j_{1}, j_{2}, \ldots, j_{k} \in[n]$, and $k \leq d\left(\epsilon^{2}\right)$. It is straightforward to see that there are such $n^{O\left(d\left(\epsilon^{2}\right)\right)}$ functions; polynomial regression runs in time polynomial in the number of basis functions.

In the remainder of the manuscript, we define noise sensitivity and prove a noise sensitivity bound for linear threshold functions over $S_{n}$ in Section 5 . The bulk of our effort is applied to showing that noise sensitivity bounds imply $\epsilon$-concentration in Section 6. We give applications to learning in Section 7.

\section{LINEAR THRESHOLD FUNCTIONS AND NOISE SENSITIVITY IN $\boldsymbol{S}_{\boldsymbol{n}}$}

We recall our definition of noise sensitivity.

Definition 5.1 (Restatement of Definition 1.1). For a function $f: S_{n} \rightarrow\{-1,1\}$ and a parameter $0<\delta<1$, we define the noise sensitivity of $f$ at $\delta$, denoted $\mathbb{N S}_{\delta}(f)$, in the following way: Let $\boldsymbol{\alpha} \sim \operatorname{Binomial}(n, \delta)$, and choose a uniformly random subset $\boldsymbol{S}$ of $[n]$ of cardinality $\alpha$. Let $\psi$ be a uniformly random permutation in $S_{n}$ such that every element of $[n] \backslash \boldsymbol{S}$ is a fixed point of $\psi$. Then

$$
\mathbb{N S}_{\delta}(f)=\underset{\sigma, \psi}{\operatorname{Pr}}[f(\boldsymbol{\sigma}) \neq f(\boldsymbol{\psi} \boldsymbol{\sigma})],
$$

where $\sigma$ is chosen uniformly at random from $S_{n}$.

To see the combinatorial parallel with the hypercube, we remark that the definition of noise sensitivity of $f$ at $\delta$ for $f:\{0,1\}^{n} \rightarrow\{-1,1\}$ can be expressed in the following way: Let $\boldsymbol{\alpha} \sim \operatorname{Binomial}(n, \delta)$, and choose a uniformly random subset $\boldsymbol{S}$ of $[n]$ of cardinality $\boldsymbol{\alpha}$. Let $\boldsymbol{y}$ be a uniformly random string in $\mathbb{Z}_{2}^{n}$ such that $\boldsymbol{y}_{i}=0$ for all $i \in[n] \backslash \boldsymbol{S}$. Then

$$
\mathbb{N S}_{\delta}(f)=\underset{\boldsymbol{x}, \boldsymbol{y}}{\mathbf{P r}}[f(\boldsymbol{x}) \neq f(\boldsymbol{x}+\boldsymbol{y})],
$$

where $\boldsymbol{x}$ is chosen uniformly at random from $\{0,1\}^{n}$, and addition is done modulo 2 .

We give a (well-studied) generalization based on random walks on Cayley graphs in Appendix A; the content there contains several references to Section 6.

\subsection{Linear Threshold Functions}

Recall the definition of linear threshold functions over $S_{n}$.

Definition 5.2. We say that a function $f: S_{n} \rightarrow\{-1,1\}$ is a linear threshold function if there exist weights $w_{i j}$ for $1 \leq i \neq j \leq n$ and a threshold $\theta$ such that $f(\sigma)=$ $\operatorname{sgn}\left(\sum_{i, j} w_{i j} \mathbf{1}[\sigma(i)=j]-\theta\right)$ for all $\sigma \in S_{n}$.

We remind the reader of Peres' Theorem in the Boolean hypercube case:

Theorem 5.3. Let $f:\{0,1\}^{n} \rightarrow\{-1,1\}$ be a linear threshold function. Then $\mathbb{N S}_{\delta}(f)=$ $O(\sqrt{\delta})$ under the uniform distribution on $\{0,1\}^{n}$. 
The notion of noise sensitivity in a product distribution is much easier to deal with, because each coordinate can be handled independently. Similarly to the argument in Blais et al. [2010], we will (nontrivially) reduce to the uniform distribution on the Boolean hypercube case.

Theorem 5.4 (Theorem 3.6). Let $f: S_{n} \rightarrow\{-1,1\}$ be a linear threshold function. Then $\mathbb{N S}_{\delta}(f) \leq \sqrt{2 \delta}$.

Proof. Our proof will closely mirror the proof of Peres' Theorem given in O'Donnell [2007]. Analogously to the proof there, we will prove a slightly stronger statement. Let $F_{1}, \ldots, F_{m}$ be any partition of $[n]$. For each $F_{i}$, let $\psi_{i}$ be an arbitrary permutation such that every point of $[n] \backslash F_{i}$ is a fixed point of $\psi_{i}$. Our strategy is to analyze the experiment where we apply one of the $\psi_{i}$ 's to $\sigma$ at random.

Lemma 5.5. Let $f: S_{n} \rightarrow\{-1,1\}$ be a linear threshold function. Let $F_{1}, \ldots, F_{m} \subseteq[n]$ be a partition of $[n]$ and let $\psi_{1}, \ldots, \psi_{m}$ be permutations as previously mentioned. Then $\operatorname{Pr}_{\sigma, i}\left[f(\boldsymbol{\sigma}) \neq f\left(\psi_{\boldsymbol{i}} \boldsymbol{\sigma}\right)\right] \leq m^{-1 / 2}$.

When $m=1 / \delta$, it is straightforward to check that the distribution on $\psi_{i} \sigma$ is the same as our previously mentioned noise experiment, when the partition and the $\psi_{i}$ 's are chosen uniformly at random as well. Every coordinate in $\sigma$ is nonfixed with probability $\delta$.

Proof of Lemma 5.5. We note that all the $\psi_{i}$ 's commute under composition. We identify every string $x \in\{0,1\}^{m}$ with the permutation $\psi_{x} \sigma$, where $\psi_{x}$ denotes the composition of the $\psi_{i}$ 's, where $x_{i}=1$. We note that $\psi_{x} \sigma$ is uniformly distributed on $S_{n}$ if $\sigma$ is. We define $g_{\sigma}:\{0,1\}^{m} \rightarrow\{-1,1\}$ such that $g_{\sigma}(x)=f\left(\psi_{x} \sigma\right)$. We have

$$
\begin{aligned}
& \underset{\boldsymbol{\sigma}, \boldsymbol{i}}{\mathbf{P r}}\left[f(\boldsymbol{\sigma}) \neq f\left(\psi_{\boldsymbol{i}} \boldsymbol{\sigma}\right)\right]=\underset{\boldsymbol{\sigma}}{\mathbf{E}}\left[\underset{\boldsymbol{i}}{\mathbf{P r}}\left[f(\boldsymbol{\sigma}) \neq f\left(\psi_{\boldsymbol{i}} \boldsymbol{\sigma}\right)\right]\right]
\end{aligned}
$$

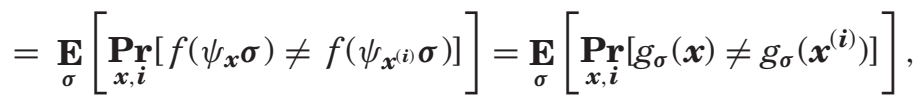

where we have used $\boldsymbol{x}^{(\boldsymbol{i})}$ to denote $\boldsymbol{x}$ with the $\boldsymbol{i}$ th bit flipped.

Consider $\mathbf{P r}_{x, i}\left[g_{\sigma}(\boldsymbol{x}) \neq g_{\sigma}\left(\boldsymbol{x}^{(i)}\right)\right]$, the quantity inside the expectation with $\sigma$ fixed to $\sigma$. Because $f$ is a linear threshold function, $g$ is also a linear threshold function in the traditional binary sense. Further,

$$
\underset{\boldsymbol{x}, \boldsymbol{i}}{\mathbf{P r}}\left[g_{\sigma}(\boldsymbol{x}) \neq g_{\sigma}\left(\boldsymbol{x}^{(\boldsymbol{i})}\right)\right]=\frac{1}{m} \operatorname{Inf}\left(g_{\sigma}\right) \leq m^{-1 / 2},
$$

using the definition of total influence and the well-known fact that $\operatorname{Inf}\left(g_{\sigma}\right) \leq \sqrt{m}$ since $g_{\sigma}$ is a linear threshold function. It follows that

$$
\underset{\sigma, i}{\mathbf{P r}}\left[f(\boldsymbol{\sigma}) \neq f\left(\psi_{\boldsymbol{i}} \boldsymbol{\sigma}\right)\right]=\underset{\sigma}{\mathbf{E}}\left[\underset{\boldsymbol{x}, \boldsymbol{i}}{\mathbf{P r}}\left[g_{\sigma}(\boldsymbol{x}) \neq g_{\sigma}\left(\boldsymbol{x}^{(\boldsymbol{i})}\right)\right]\right] \leq \underset{\boldsymbol{\sigma}}{\mathbf{E}}\left[m^{-1 / 2}\right]=m^{-1 / 2},
$$

finishing the proof of Lemma 5.5.

To complete the proof of the theorem, we notice that the noise experiment can be thought of in the following way, when $\delta=1 / m$ for an integer $m$ : For each $i \in[n]$, put $i$ in one of $\boldsymbol{F}_{1}, \ldots, \boldsymbol{F}_{m}$ uniformly at random. Every coordinate is in any $\boldsymbol{F}_{j}$ with probability $\delta=1 / \mathrm{m}$. We randomly choose permutations $\psi_{j}$ where $j \in[\mathrm{m}]$ such that every point in $[n] \backslash \boldsymbol{F}_{j}$ is fixed by $\boldsymbol{\psi}_{j}$.

Using the lemma:

$$
\mathbb{N S}_{\delta}(f)=\mathbf{E}_{\boldsymbol{F}_{1}, \ldots, \boldsymbol{F}_{m}, \boldsymbol{\psi}_{1}, \ldots, \boldsymbol{\psi}_{m}}\left[\mathbf{P r}_{\boldsymbol{\sigma}, i}\left[f(\boldsymbol{\sigma}) \neq f\left(\boldsymbol{\psi}_{\boldsymbol{i}} \boldsymbol{\sigma}\right)\right]\right] \leq m^{-1 / 2}=\sqrt{\delta} .
$$


If $\delta$ is not the reciprocal of an integer, then we use the fact that noise sensitivity is increasing in $\delta$, and round up to the nearest reciprocal of an integer $\delta^{\prime}$. We have $\delta^{\prime} \leq 2 \delta$, so we have

$$
\mathbb{N S}_{\delta}(f) \leq \mathbb{N S}_{\delta^{\prime}}(f) \leq \sqrt{\delta^{\prime}} \leq \sqrt{2 \delta}
$$

completing the proof.

\section{NOISE SENSITIVITY AND FOURIER ANALYSIS}

In this section, we will interpret the noise sensitivity of a function over $S_{n}$ in terms of its Fourier spectrum. We remind the reader of Definition 5.1 here. For $0 \leq \delta \leq 1$, we define the distribution $N_{\delta}$ to be the distribution over $S_{n}$ whose samples can be generated as follows: select $\boldsymbol{k} \sim \operatorname{Binomial}(n, \delta)$, and a uniformly random subset $\boldsymbol{S} \subseteq[n]$ of cardinality $\boldsymbol{k}$. The sample is a uniformly random permutation such that every coordinate outside of $\boldsymbol{S}$ is a fixed point of the permutation. In the context of Definition 5.1, $\psi$ is distributed as $N_{\delta}$.

\subsection{Operators}

Definition 6.1. For $0 \leq \delta \leq 1$, we define the functional operator $T_{\delta}$ such that, given a function $f: S_{n} \rightarrow \mathbb{R}$, the function $T_{\delta} f$ is defined such that

$$
\left(T_{\delta} f\right)(\sigma)=\underset{\psi \sim N_{\delta}}{\mathbf{E}}[f(\boldsymbol{\psi} \sigma)]=\underset{\psi \sim N_{\delta}}{\mathbf{E}}[f(\sigma \psi)] .
$$

The last equality above uses the fact the distribution on $N_{\delta}$ is a class distribution; $\mathbf{P r}_{\psi \sim N_{\delta}}[\psi=\tau]=\mathbf{P r}_{\psi \sim N_{\delta}}\left[\boldsymbol{\psi}=\sigma \tau \sigma^{-1}\right]$ for all $0 \leq \delta \leq 1$ and all $\tau, \sigma \in S_{n}$. For $f$ : $S_{n} \rightarrow\{-1,1\}$, we have $\mathbb{N S}_{\delta}(f)=\mathbf{P r}_{\sigma, \psi \sim N_{\delta}}[f(\sigma) \neq \bar{f}(\sigma \psi)]$. It is not hard to see that for $f: S_{n} \rightarrow\{-1,1\}$, we have

$$
\mathbb{N S}_{\delta}(f)=\frac{1}{2}-\frac{1}{2} \underset{\sigma, \psi \sim N_{\delta}}{\mathbf{E}}[f(\sigma) f(\sigma \psi)]=\frac{1}{2}-\frac{1}{2}\left\langle f, T_{\delta} f\right\rangle
$$

As might be expected, we are aiming to use the Fourier interpretation of noise. For analysis purposes, one critical fact is that $N_{\delta}$ is a class distribution; that is, the probability distribution is uniform given a fixed conjugacy class, so this operator is diagonalized by the Fourier transform in a strong way.

LEMma 6.2. Let $U$ be any functional operator operating on functions $f: S_{n} \rightarrow \mathbb{R}$, where $U f$ is such that $U f(\sigma)=\mathbf{E}_{\psi}[f(\psi \sigma)]$ for some random variable $\psi$. If the distribution on $\psi$ is a class distribution, and $\left\{\widehat{f}_{\lambda}\right\}$ is the Fourier spectrum of $f$, then for every $\lambda \vdash n$,

$$
\widehat{U f}_{\lambda}=k_{\lambda} \widehat{f}_{\lambda} \quad \text { where } \quad k_{\lambda}=\frac{\operatorname{tr} \mathbf{E}_{\psi}\left[\rho_{\lambda}(\psi)\right]}{\operatorname{dim} \lambda},
$$

where $\operatorname{tr}$ denotes the trace of a matrix. Note that $k_{\lambda}$ is independent of $f$.

PRoof. This fact essentially follows from Schur's representation lemma (for example, see James and Kerber [1981]), but we give a proof here for completeness. Let $\rho$ be any irreducible representation corresponding to the partition $\lambda$. Since $U$ is a linear operator, it suffices to show that for any function $\rho_{i j}$ given by an $(i, j)$-entry of an irreducible representation $\rho$, we have

$$
U \rho_{i j}=k_{\rho} \rho_{i j}
$$


for some constant $k_{\rho}$. Starting from the definition of $U$ and using the fact that $\rho$ is a representation, we get

$$
\begin{aligned}
\left(U \rho_{i j}\right)(\sigma) & =\underset{\psi}{\mathbf{E}}\left[\rho_{i j}(\sigma \boldsymbol{\psi})\right] \\
& =\underset{\psi}{\mathbf{E}}\left[(\rho(\sigma) \rho(\boldsymbol{\psi}))_{i j}\right] \\
& =\underset{\psi}{\mathbf{E}}\left[\sum_{k=1}^{d_{\rho}} \rho(\sigma)_{i k} \rho(\boldsymbol{\psi})_{k j}\right] \\
& =\sum_{k=1}^{d_{\rho}} \rho(\sigma)_{i k} \underset{\psi}{\mathbf{E}}\left[\rho(\boldsymbol{\psi})_{k j}\right] \\
& =\sum_{k=1}^{d_{\rho}} \rho(\sigma)_{i k}(\underset{\psi}{\mathbf{E}}[\rho(\boldsymbol{\psi})])_{k j} \\
& =(\rho(\sigma) \cdot \underset{\psi}{\mathbf{E}}[\rho(\boldsymbol{\psi})])_{i j}
\end{aligned}
$$

for any $i$ and $j$. By symmetry and the fact that $\psi$ is a class distribution, we also have

$$
\left.\left(U \rho_{i j}\right)(\sigma)=\underset{\psi}{\mathbf{E}}\left[\rho_{i j}(\boldsymbol{\psi} \sigma)\right]=\underset{\psi}{\mathbf{E}}[\rho(\boldsymbol{\psi})] \cdot \rho(\sigma)\right)_{i j}
$$

for any $i$ and $j$. Thus, $\rho(\sigma)$ and $\mathbf{E}_{\psi}[\rho(\psi)]$ commute for any $\sigma$. Because $\rho$ was taken to be irreducible, the set of matrices $\left\{\rho(\sigma): \sigma \in S_{n}\right\}$ is a basis for the vector space of square matrices of size $d_{\rho}$. It follows that $\mathbf{E}_{\psi}[\rho(\psi)]$ commutes with every square matrix of size $d_{\rho}$. This is sufficient to conclude that $\mathbf{E}_{\psi}[\rho(\boldsymbol{\psi})]=k_{\rho} I_{d_{\rho}}$ for some constant $k_{\rho}$, and thus

$$
\left(U \rho_{i j}\right)(\sigma)=\left(\rho(\sigma) \cdot k_{\rho} I_{d_{\rho}}\right)_{i j}=k_{\rho} \rho_{i j}(\sigma) .
$$

The claim follows by applying the linearity of $U$. Further, $\operatorname{tr} \mathbf{E}_{\psi}\left[\rho_{\lambda}(\boldsymbol{\psi})\right]=(\operatorname{dim} \lambda) k_{\rho_{\lambda}}$, yielding the expression for $k_{\rho_{\lambda}}$ in the statement of the lemma.

Corollary 6.3. For any positive integer $n$ and $0<\delta<1$ there exist constants $c_{\lambda, \delta}$ and $c_{\lambda, \delta}^{\prime}$ such that for every $f: S_{n} \rightarrow\{-1,1\}$, we have

$$
\left\langle f, T_{\delta} f\right\rangle=\sum_{\lambda \vdash n} c_{\lambda, \delta}^{\prime}\left\|\widehat{f}_{\lambda}\right\|^{2} d_{\lambda} \quad \text { and } \quad \mathbb{N S}_{\delta}(f)=\sum_{\lambda \vdash n} c_{\lambda, \delta}\left\|\widehat{f_{\lambda}}\right\|^{2} d_{\lambda},
$$

where

$$
c_{\lambda, \delta}^{\prime}=\frac{\operatorname{tr} \mathbf{E}_{\psi \sim N_{\delta}}\left[\rho_{\lambda}(\boldsymbol{\psi})\right]}{\operatorname{dim} \lambda} \quad \text { and } \quad c_{\lambda, \delta}=\frac{1}{2}-\frac{1}{2} c_{\lambda, \delta}^{\prime}
$$

Proof. By the previous lemma, we have $\widehat{T_{\delta} f_{\lambda}}=c_{\lambda, \delta}^{\prime} \hat{f}_{\lambda}$ for $\lambda \vdash n$, and $c_{\lambda, \delta}^{\prime}=$ $\mathbf{E}_{\psi \sim N_{\delta}}\left[\rho_{\lambda}(\boldsymbol{\psi})\right]$. The first equation follows from applying Plancherel's identity, and the second follows easily from the first since $\mathbb{N S}_{\delta}(f)=\frac{1}{2}-\frac{1}{2}\left\langle f, T_{\delta} f\right\rangle$.

\subsection{Characters}

In order to show that low noise sensitivity implies some level of concentration, we will derive expressions for the constants $c_{\lambda, \delta}$ and $c_{\lambda, \delta}^{\prime}$ in Corollary 6.3. We will first consider the distribution $N_{\delta}$ conditioned on the number of coordinates specified to be fixed points (which has distribution $\operatorname{Binomial}(n, 1-\delta)$ ). Specifically, we define $W_{k}$ to be the distribution over $S_{n}$ whose samples can be generated in the following way: Uniformly randomly select $n-k$ of the $n$ letters to be guaranteed to be fixed points, and select a 
random permutation over the other $k$ elements. The noise distribution $N_{\delta}$ is a mixture of $W_{k}$ distributions; a draw from $N_{\delta}$ is distributed the same as a draw from $W_{k}$, where $\boldsymbol{k} \sim \operatorname{Binomial}(n, \delta)$.

To analyze $c_{\lambda, \delta}^{\prime}$, we consider $\operatorname{tr} \mathbf{E}_{\psi \sim N_{\delta}}[\rho(\boldsymbol{\psi})]$. It is enough to determine $\operatorname{tr} \mathbf{E}_{\psi \sim W_{k}}[\rho(\boldsymbol{\psi})]$ for every $\rho$. Recall that the character $\chi_{\rho}$ of a representation $\rho$ is the function defined by $\chi_{\rho}(\sigma)=\operatorname{tr} \rho(\sigma)$. The character of all representations corresponding to the same partition yield the same character, so $\chi_{\rho}$ is well defined. It is not hard to see that representations (characters) of $G$ are also representations (characters) of any subgroup of $G$, although an irreducible representation of $G$ might not be an irreducible representation for every subgroup. For a character $\chi$ of a group $G$ and a subgroup $H \leq G$, we define $\operatorname{Res}_{H} \chi$ to be the character restricted to $H$.

We first note that

$$
\begin{aligned}
\operatorname{tr} \underset{\psi \sim W_{k}}{\mathbf{E}}[\rho(\boldsymbol{\psi})] & =\underset{\psi \sim W_{k}}{\mathbf{E}}[\operatorname{tr} \rho(\boldsymbol{\psi})] \\
& =\underset{\psi \sim W_{k}}{\mathbf{E}}\left[\chi_{\rho}(\boldsymbol{\psi})\right] .
\end{aligned}
$$

The character function is a class function on $S_{n}$, so which $n-k$ points are fixed does not matter. We will assume that the fixed points are $\{k+1, k+2, \ldots, n\}$. We can ignore these fixed points and treat $\psi$ as a uniformly chosen element of $S_{k}$, and thus

$$
\underset{\psi \sim W_{k}}{\mathbf{E}}\left[\chi_{\rho}(\boldsymbol{\psi})\right]=\underset{\psi \sim S_{k}}{\mathbf{E}}\left[\operatorname{Res}_{S_{k}} \chi_{\rho}(\boldsymbol{\psi})\right]
$$

Let $\mu=(k)$. The corresponding representation is the trivial representation (which we will denote triv), whose character $\chi_{\text {triv }}$ is the constant function 1 . Thus,

$$
\underset{\psi \sim S_{k}}{\mathbf{E}}\left[\operatorname{Res}_{S_{k}} \chi_{\rho}(\boldsymbol{\psi})\right]=\underset{\psi \sim S_{k}}{\mathbf{E}}\left[\operatorname{Res}_{S_{k}} \chi_{\rho}(\boldsymbol{\psi}) \operatorname{Res}_{S_{k}} \chi_{t r i v}(\boldsymbol{\psi})\right]=\left\langle\operatorname{Res}_{S_{k}} \chi_{\rho}, \operatorname{Res}_{S_{k}} \chi_{t r i v}\right\rangle,
$$

where the inner product is taken in $S_{k}$. It is known (see, for example, (8.1) in Okounkov and Olshanski [1998]) that

$$
\left\langle\operatorname{Res}_{S_{k}} \chi_{\rho}, \operatorname{Res}_{S_{k}} \chi_{t r i v}\right\rangle=\operatorname{dim} \lambda /(k),
$$

where $\lambda$ is the partition corresponding to the irreducible representation $\rho$. It follows that $\operatorname{tr} \mathbf{E}_{\psi \sim W_{k}}[\rho(\boldsymbol{\psi})]=\operatorname{dim} \lambda /(k)$. Using the fact that $N_{\delta}$ is a mixture of distributions of the form $W_{k}$ and applying Corollary 6.3, we get:

Proposition 6.4. $c_{\lambda, \delta}^{\prime}=\mathbf{E}_{\boldsymbol{k}}\left[\frac{\operatorname{dim} \lambda /(\boldsymbol{k})}{\operatorname{dim} \lambda}\right]$, where the distribution on $\boldsymbol{k}$ is $\operatorname{Binomial}(n, \delta)$.

We will make use of an expression for $\frac{\operatorname{dim} \lambda /(k)}{\operatorname{dim} \lambda}$ due to Okounkov and Olshanski [1998]. They actually give a much more general statement in (8.3) of their article:

$$
\frac{\operatorname{dim} \lambda / \mu}{\operatorname{dim} \lambda}=\frac{(n-k) !}{n !} s_{\mu}^{*}(\lambda)
$$

for any partition $\mu \vdash k$. The expression $s_{\mu}^{*}(\lambda)$ is a shifted Schur function. While the definition of shifted Schur functions is cumbersome, Okounkov and Olshanski [1998] give a handy interpretation of these shifted Schur functions in terms of tableaux. For our purposes, we only need the case where $\mu=(k)$; in this case, applying their Theorem 11.1, we get

$$
s_{(k)}^{*}\left(x_{1}, x_{2}, \ldots\right)=\sum_{1 \leq i_{1} \leq i_{2} \cdots \leq i_{k}<\infty}\left(x_{i_{1}}-k+1\right)\left(x_{i_{2}}-k+2\right) \cdots x_{i_{k}} .
$$


In computing $s_{(k)}^{*}(\lambda)$, we imagine that $\lambda$ is appended with infinitely many zeroes. If $\lambda=\left(\lambda_{1}, \lambda_{2}, \ldots, \lambda_{\ell}\right)$, then the terms in the sum can only be positive if $i_{k} \leq \ell$, and none of the terms are negative.

\subsection{Dominance Order}

Recall that the dominance ordering on partitions is the partial order such that $\lambda \unrhd \beta$ if $\sum_{i=1}^{k} \lambda_{i} \geq \sum_{i=1}^{k} \beta_{i}$ for all $k \geq 1$. Intuitively, the higher degree representations should contribute more to the noise sensitivity; this is indeed the case. To show this, we prove the following result, which might be of independent interest (we remark that this was proved for $k=2$ in Diaconis [1988]):

Theorem 6.5. Let $\lambda \vdash n$ and $\beta \vdash n$ such that $\lambda \unrhd \beta$. Then

$$
\frac{\operatorname{dim} \lambda /(k)}{\operatorname{dim} \lambda} \geq \frac{\operatorname{dim} \beta /(k)}{\operatorname{dim} \beta}
$$

for any integer $k \geq 0$.

Proof. It suffices to consider the case that $\lambda \vdash n$ and $\beta \vdash n$ are such that $\lambda \unrhd \beta$ and there does not exist a partition $\alpha \vdash n$ such that $\alpha \notin\{\lambda, \beta\}$ and $\lambda \unrhd \alpha \unrhd \beta$. In this case, there exist indices $s<t$ such that $\lambda_{s}=\beta_{s}+1, \lambda_{t}=\beta_{t}-1, \lambda_{j}=\beta_{j}$ for $s<j<t$ (this interval might be empty), and $\beta_{j}$ is constant for $s \leq j \leq t$. Using Equation (1), it suffices to show that $s_{(k)}^{*}\left(\lambda_{1}, \lambda_{2}, \ldots, \lambda_{\ell}\right) \geq s_{(k)}^{*}\left(\beta_{1}, \beta_{2}, \ldots, \beta_{\ell}\right)$; we will do so using the expression in Equation (2). We remark that the structure of these expressions suggests that perhaps we could show this using some general purpose inequality (such as something similar to the Karamata Inequality [Karamata 1932]), but we were unable to find a suitable inequality in the literature.

We prove the statement by induction. For the case $k=0$, we see that $s_{(0)}^{*}$ is an empty product and thus identically 1 .

For the inductive step, we decompose the sum in Equation (2) into two separate sums over sequences where $i_{1}=1$ and $i_{1} \neq 1$; we get

$$
s_{(k)}^{*}(\lambda)=s_{(k)}^{*}\left(\lambda_{1}, \lambda_{2}, \ldots, \lambda_{\ell}\right)=\left(\lambda_{1}-k+1\right) s_{(k-1)}^{*}\left(\lambda_{1}, \lambda_{2}, \lambda_{3}, \ldots, \lambda_{\ell}\right)+s_{(k)}^{*}\left(\lambda_{2}, \lambda_{3}, \ldots, \lambda_{\ell}\right),
$$

and similarly for $s_{(k)}^{*}(\beta)$. If $\lambda_{1}=\beta_{1}$, then $\left(\lambda_{2}, \lambda_{3}, \ldots, \lambda_{\ell}\right) \unrhd\left(\beta_{2}, \beta_{3}, \ldots, \beta_{\ell}\right)$ and $\lambda_{1}-k+1=$ $\beta_{1}-k+1$, and we are done by induction.

Similarly, separately summing over sequences where $i_{k}=\ell$ and $i_{k} \neq \ell$, we get

$$
s_{(k)}^{*}(\lambda)=s_{(k-1)}^{*}\left(\lambda_{1}-1, \lambda_{2}-1, \ldots, \lambda_{\ell-1}-1, \lambda_{\ell}-1\right) \lambda_{\ell}+s_{(k)}^{*}\left(\lambda_{1}, \lambda_{2}, \ldots, \lambda_{\ell-1}\right)
$$

and similarly for $\beta$. If $\lambda_{\ell}=\beta_{\ell}$, then we are done by induction again using a similar argument as above. Thus, without loss of generality, we can assume that $\lambda_{1} \neq \beta_{1}$ and $\lambda_{\ell} \neq \beta_{\ell}$; this implies $\lambda_{1}=\beta_{1}+1, \lambda_{\ell}=\beta_{\ell}-1$, and $\lambda_{2}=\lambda_{3}=\cdots=\lambda_{\ell-1}=\beta_{2}=\beta_{3}=\cdots=$ $\beta_{\ell-1}$.

Let $\operatorname{seq}_{k, \ell}$ be the set of nondecreasing sequences $\left\{i_{1}, \ldots, i_{k}\right\}$ where $1 \leq i_{j} \leq \ell$. For $i \in$ $\operatorname{seq}_{k, \ell}$, we define the function $\pi_{i}: \mathbb{R}^{k} \rightarrow \mathbb{R}$ such that $\pi_{i}(x)=\left(x_{i_{1}}-k+1\right)\left(x_{i_{2}}-k+2\right) \cdots x_{i_{k}}$. We allow $i$ to be an empty sequence and define $\pi_{i}(x) \equiv 1$ in this case. By decomposing the sum, we have reduced the problem to showing

$$
\sum_{i \in \mathrm{Seq}_{k, \ell}} \pi_{i}(\lambda) \geq \sum_{i \in \mathrm{Seq}_{k, \ell}} \pi_{i}(\beta) .
$$

For a nondecreasing sequence $i \in \operatorname{seq}_{k, \ell}$, let $\operatorname{revcmp}(i)$ (short for "reverse complement") be the sequence such that $\operatorname{revcmp}(i)_{j}=\ell+1-i_{k+1-j}$. To prove Inequality (3), 
it suffices to prove

$$
\pi_{i}(\lambda)+\pi_{\operatorname{revcmp}(i)}(\lambda) \geq \pi_{i}(\beta)+\pi_{\operatorname{revcmp}(i)}(\beta)
$$

for all $i \in \operatorname{seq}_{k, \ell} ;$ Inequality (3) follows by summing Inequality (4) over pairs of sequences $\{i, \operatorname{revcmp}(i)\}$. (Note that $i \mapsto \operatorname{revcmp}(i)$ is a bijection, $i=\operatorname{revcmp}(\operatorname{revcmp}(i))$, and for some sequences $i=\operatorname{revcmp}(i))$. Because $\lambda_{i}$ and $\beta_{i}$ only take the three values $\left\{\beta_{1}-\right.$ $\left.1, \beta_{1}, \beta_{1}+1\right\}$, we define

$$
\begin{aligned}
\pi_{a, b, c}\left(\beta_{1}\right) & =\left(\left(\beta_{1}+1\right)-k+1\right)^{(a)}\left(\beta_{1}-(k-a)+1\right)^{(b)}\left(\left(\beta_{1}-1\right)-(k-a-b)+1\right)^{(c)} \\
& =\left(\beta_{1}-k+2\right)^{(a)}\left(\beta_{1}-k+a+1\right)^{(b)}\left(\left(\beta_{1}-k+a+b\right)^{(c)},\right.
\end{aligned}
$$

where $k=a+b+c$ for nonnegative integers $a, b$, and $c$, and $(x)^{(y)}$ denotes the rising factorial function; $(x)^{(y)}=x(x+1)(x+2) \cdots(x+y-1)$. The quantity $\pi_{a, b, c}\left(\beta_{1}\right)$ is exactly $\pi_{i}(\lambda)$ when $i$ is a sequence of indices starting with $a$ values of 1 and ending with $c$ values of $\ell$, with $b$ other values in between. We will frequently relate quantities of this form to each other; for example, we have the following identities by "peeling" terms off the end of the outer rising factorials:

$$
\begin{gathered}
\pi_{a, b, c}\left(\beta_{1}\right)=\left(\beta_{1}-k+2\right) \cdot \pi_{a-1, b, c}\left(\beta_{1}\right)=\pi_{a, b, c-1}\left(\beta_{1}-1\right) \cdot\left(\beta_{1}-1\right) \\
\pi_{0, b, c}\left(\beta_{1}\right)=\left(\beta_{1}-k+1\right) \cdot \pi_{0, b-1, c}\left(\beta_{1}\right) \quad \pi_{a, b, 0}\left(\beta_{1}\right)=\pi_{a, b-1,0}\left(\beta_{1}-1\right) \cdot \beta_{1} .
\end{gathered}
$$

Now $\pi_{i}(\beta)=\pi_{\operatorname{revcmp}(i)}(\beta)=\pi_{0, k, 0}\left(\beta_{1}\right)$, since $\beta_{1}=\cdots=\beta_{\ell}$. Then $\operatorname{revcmp}(i)$ begins with $c$ values of 1 and ends with $a$ values of $\ell$, so to show Inequality (4) we are tasked with showing

$$
\pi_{a, b, c}\left(\beta_{1}\right)+\pi_{c, b, a}\left(\beta_{1}\right) \geq 2 \pi_{0, k, 0}\left(\beta_{1}\right),
$$

where $a, b$, and $c$ are nonnegative and $a+b+c=k \leq \beta_{1}$. We will prove this by induction on $k$. If $a=0$ and $c=0$, then Inequality (5) holds with equality. If $a \neq 0$ and $c \neq 0$, then $k \geq 2$, and we have

$$
\begin{aligned}
\pi_{a, b, c}\left(\beta_{1}\right)+\pi_{c, b, a}\left(\beta_{1}\right) & =\left(\beta_{1}-k+2\right)\left(\pi_{a-1, b, c-1}\left(\beta_{1}-1\right)+\pi_{c-1, b, a-1}\left(\beta_{1}-1\right)\right)\left(\beta_{1}-1\right) \\
& \geq\left(\beta_{1}-k+1\right)\left(\pi_{a-1, b, c-1}\left(\beta_{1}-1\right)+\pi_{c-1, b, a-1}\left(\beta_{1}-1\right)\right)\left(\beta_{1}\right) \\
& \geq\left(\beta_{1}-k+1\right)\left(2 \pi_{0, k-2,0}\left(\beta_{1}-1\right)\right)\left(\beta_{1}\right) \\
& =2 \pi_{0, k, 0}\left(\beta_{1}\right),
\end{aligned}
$$

where we used the fact that $k \leq \beta_{1}$ in the first inequality and induction in the second. For the remaining cases, assume without loss of generality that $a=0$ and $c \neq 0$. If $b=0$, then $c=k$, and we have $\pi_{0,0, c}\left(\beta_{1}\right)+\pi_{c, 0,0}\left(\beta_{1}\right)=\left(\beta_{1}-c\right)^{(c)}+\left(\beta_{1}-c+2\right)^{(c)} \geq$ $2\left(\beta_{1}-c+1\right)^{(c)}=2 \pi_{0, c, 0}\left(\beta_{1}\right)$, using the convexity of the rising factorial function for positive arguments in the inequality. (This argument also completes the base case $k=1$.) In the rest of the proof, we assume $b \neq 0$. We have

$$
\pi_{0, b, c}\left(\beta_{1}\right)+\pi_{c, b, 0}\left(\beta_{1}\right)=\pi_{0, b, c-1}\left(\beta_{1}-1\right) \cdot\left(\beta_{1}-1\right)+\left(\beta_{1}-k+2\right) \cdot \pi_{c-1, b, 0}\left(\beta_{1}\right) .
$$

Note that each of $\pi_{0, b, c-1}\left(\beta_{1}-1\right)$ and $\pi_{c-1, b, 0}\left(\beta_{1}\right)$ can be written as a product of a sequence of $k-1$ nondecreasing nonnegative integers if we expand the rising factorials in the natural way. The first integer in the product for $\pi_{0, b, c-1}\left(\beta_{1}-1\right)$ is $\beta_{1}-1-$ $(k-1)+1=\beta_{1}-k+1$, where the first integer in the product for $\pi_{c-1, b, 0}\left(\beta_{1}\right)$ is $\beta_{1}+$ $1-(k-1)+1=\beta_{1}-k+3$. In each of these sequences, the next integer is 1 more than the preceding integer, save for one possible case where two consecutive integers are the same. Since the corresponding integers start with a difference of 2 , the $j$ th multiplier in the product for $\pi_{0, b, c-1}\left(\beta_{1}-1\right)$ is at most the $j$ th multiplier in the product 
for $\pi_{c-1, b, 0}\left(\beta_{1}\right)$. Thus $\pi_{0, b, c-1}\left(\beta_{1}-1\right) \leq \pi_{c-1, b, 0}\left(\beta_{1}\right)$. We now have

$$
\begin{aligned}
\pi_{0, b, c}\left(\beta_{1}\right)+\pi_{c, b, 0}\left(\beta_{1}\right)= & \pi_{0, b, c-1}\left(\beta_{1}-1\right) \cdot\left(\beta_{1}-1\right)+\left(\beta_{1}-k+2\right) \cdot \pi_{c-1, b, 0}\left(\beta_{1}\right) \\
\geq & \pi_{0, b, c-1}\left(\beta_{1}-1\right) \cdot\left(\beta_{1}\right)+\left(\beta_{1}-k+1\right) \cdot \pi_{c-1, b, 0}\left(\beta_{1}\right) \\
= & \left(\beta_{1}-k+1\right) \cdot \pi_{0, b-1, c-1}\left(\beta_{1}-1\right) \cdot\left(\beta_{1}\right) \\
& +\left(\beta_{1}-k+1\right) \cdot \pi_{c-1, b-1,0}\left(\beta_{1}-1\right) \cdot\left(\beta_{1}\right) \\
= & \left(\beta_{1}-k+1\right) \cdot\left(\pi_{0, b-1, c-1}\left(\beta_{1}-1\right)+\pi_{c-1, b-1,0}\left(\beta_{1}-1\right)\right) \cdot\left(\beta_{1}\right) \\
\geq & \left(\beta_{1}-k+1\right) \cdot\left(2 \pi_{0, k-2,0}\left(\beta_{1}-1\right)\right) \cdot\left(\beta_{1}\right) \\
= & 2 \pi_{0, k, 0}\left(\beta_{1}\right),
\end{aligned}
$$

using induction for the second inequality.

This theorem is, in general, false if we allow partitions other than $(k)$ for $\mu$. For example, one can check that $(4,2) \unrhd(3,2,1), \operatorname{dim}(4,2)=9, \operatorname{dim}(3,2,1)=16$, $\operatorname{dim}(4,2) /(2,1)=3$, and $\operatorname{dim}(3,2,1) /(2,1)=6$, but $3 / 9=1 / 3<3 / 8=6 / 16$.

Corollary 6.6. Let $S=\left\{\lambda \vdash n: \lambda_{1} \leq n-\ell\right\}$ for some $\ell$. For every $0 \leq \delta \leq 1$, the maximum of $c_{\lambda, \delta}^{\prime}$ for $\lambda \in S$ occurs when $\lambda=(n-\ell, \ell)$.

Proof. For every $\lambda \in S$, we have $c_{\lambda, \delta}^{\prime}=\mathbf{E}\left[\frac{\operatorname{dim} \lambda /(\boldsymbol{k})}{\operatorname{dim} \lambda}\right]$. We apply the previous theorem for each $0 \leq k \leq n$, using the fact that $(n-\ell, \ell) \unrhd \lambda$ for all $\lambda \in S$. The result follows by taking the expectation.

\subsection{Concentration}

The results of the previous section tell us that as a partition gets more "stretched out," the corresponding representation is more sensitive to noise. We show that $\frac{\operatorname{dim} \lambda /(k)}{\operatorname{dim} \lambda}$ is upper bounded by an expression exponentially decreasing in $k$; we encourage the reader to compare to the hypercube case. It suffices to analyze partitions of $n$ into two parts: $\lambda=\left(\lambda_{1}, \lambda_{2}\right) \vdash n$. From Diaconis [1988], $\frac{\operatorname{dim} \lambda /(2)}{\operatorname{dim} \lambda}$ is simple to compute directly:

$$
\frac{\operatorname{dim} \lambda /(2)}{\operatorname{dim} \lambda}=\frac{\lambda_{1}\left(\lambda_{1}-1\right)+\lambda_{2}\left(\lambda_{1}-1\right)+\lambda_{2}\left(\lambda_{2}-1\right)}{n(n-1)}=\frac{\lambda_{1}-1}{n-1}+\frac{\lambda_{2}\left(\lambda_{2}-1\right)}{n(n-1)} .
$$

Let $(a)_{b}$ be the falling factorial function; $(a)_{b}=a(a-1) \cdots(a-b+1)$. It follows from Equations (1) and (2) [Okounkov and Olshanski 1998] that, in this case, we have

$$
\frac{\operatorname{dim} \lambda /(k)}{\operatorname{dim} \lambda}=\sum_{i=0}^{k} \frac{\left(\lambda_{1}-i\right)_{k-i}\left(\lambda_{2}\right)_{i}}{(n)_{k}}
$$

With this in hand, we show that this quantity is decreasing exponentially in $k$ :

Proposition 6.7. For $\lambda=\left(\lambda_{1}, \lambda_{2}\right) \vdash n$, we have $\frac{\operatorname{dim} \lambda /(k)}{\operatorname{dim} \lambda} \leq\left(\frac{\operatorname{dim} \lambda /(2)}{\operatorname{dim} \lambda}\right)^{k-1}$.

Proof. The cases $k=0$ and $k=1$ are immediate. When $k \geq 2$, it suffices to show

$$
\frac{\operatorname{dim} \lambda /(k)}{\operatorname{dim} \lambda} \leq \frac{\operatorname{dim} \lambda /(k-1)}{\operatorname{dim} \lambda} \cdot \frac{\operatorname{dim} \lambda /(2)}{\operatorname{dim} \lambda} .
$$

It follows from Equation (7) that

$$
\frac{\operatorname{dim} \lambda /(k)}{\operatorname{dim} \lambda}=\frac{\operatorname{dim} \lambda /(k-1)}{\operatorname{dim} \lambda} \frac{\lambda_{1}-k+1}{n-k+1}+\frac{\left(\lambda_{2}\right)_{k}}{(n)_{k}} .
$$


Now, for $k \geq 2$, we have $\frac{\lambda_{1}-k+1}{n-k+1} \leq \frac{\lambda_{1}-1}{n-1}$. We will show

$\frac{\left(\lambda_{2}\right)_{k}}{(n)_{k}} \leq \frac{\operatorname{dim} \lambda /(k-1)}{\operatorname{dim} \lambda} \frac{\lambda_{2}\left(\lambda_{2}-1\right)}{n(n-1)} \quad$ or equivalently $\quad \frac{\left(\lambda_{2}-2\right)_{k-2}}{(n-2)_{k-2}} \leq \frac{\operatorname{dim} \lambda /(k-1)}{\operatorname{dim} \lambda}$.

(If $\lambda_{2}<k$, then the left-hand side of the first inequality is zero and we are done.) Indeed,

$$
\begin{aligned}
\frac{\left(\lambda_{2}-2\right)_{k-2}}{(n-2)_{k-2}} \leq \frac{\left(\lambda_{2}-1\right)_{k-2}}{(n-1)_{k-2}} & =\frac{\lambda_{1}\left(\lambda_{2}-1\right)_{k-2}}{n(n-1)_{k-2}}+\frac{\lambda_{2}\left(\lambda_{2}-1\right)_{k-2}}{n(n-1)_{k-2}} \\
& \leq \frac{\lambda_{1}\left(\lambda_{1}-1\right)_{k-2}}{(n)_{k-1}}+\frac{\lambda_{2}\left(\lambda_{2}-1\right)_{k-2}}{(n)_{k-1}}=\frac{\left(\lambda_{1}\right)_{k-1}}{(n)_{k-1}}+\frac{\left(\lambda_{2}\right)_{k-1}}{(n)_{k-1}} \\
& \leq \frac{\operatorname{dim} \lambda /(k-1)}{\operatorname{dim} \lambda},
\end{aligned}
$$

where the last inequality uses the fact that the left-hand side consists of two terms of the summation in Equation (7), and all of the terms in the summation are nonnegative. It follows that

$$
\begin{aligned}
\frac{\operatorname{dim} \lambda /(k)}{\operatorname{dim} \lambda} & =\frac{\operatorname{dim} \lambda /(k-1)}{\operatorname{dim} \lambda} \frac{\lambda_{1}-k+1}{n-k+1}+\frac{\left(\lambda_{2}\right)_{k}}{(n)_{k}} \\
& \leq \frac{\operatorname{dim} \lambda /(k-1)}{\operatorname{dim} \lambda}\left(\frac{\lambda_{1}-1}{n-1}+\frac{\lambda_{2}\left(\lambda_{2}-1\right)}{n(n-1)}\right) \\
& =\frac{\operatorname{dim} \lambda /(k-1)}{\operatorname{dim} \lambda} \cdot \frac{\operatorname{dim} \lambda /(2)}{\operatorname{dim} \lambda},
\end{aligned}
$$

finishing the proof of the claim.

Corollary 6.8. For a partition $\lambda$ into two parts, $c_{\lambda, \delta}^{\prime} \leq \mathbf{E}\left[\left(\frac{\operatorname{dim} \lambda /(2)}{\operatorname{dim} \lambda}\right)^{\boldsymbol{k}-1}\right]$, where $\boldsymbol{k}$ is distributed as Binomial(n, $\delta$ ).

We are now ready to prove our noise sensitivity theorem:

Theorem 6.9. Suppose $f: S_{n} \rightarrow\{-1,1\}$ is such that $\mathbb{N S}_{\delta}(f) \leq \epsilon$ for some $\delta$. Then

$$
\sum_{\lambda: \lambda_{1} \leq n-10 / \delta} d_{\lambda}\left\|\widehat{f}_{\lambda}\right\|^{2} \leq \frac{2}{1-2 e^{-0.9}} \epsilon
$$

and $f$ is $O(\epsilon)$-concentrated to degree $10 / \delta$.

Proof. If $\delta \leq 10 / n$, then the statement becomes trivial, so we henceforth assume that $\delta>10 / n$ and $n>10$. Recall that

$$
\mathbb{N S}_{\delta}(f)=\sum_{\lambda}\left(\frac{1}{2}-\frac{1}{2} c_{\lambda, \delta}^{\prime}\right) d_{\lambda}\left\|\widehat{f}_{\lambda}\right\|^{2} .=\frac{1}{2}-\frac{1}{2} \sum_{\lambda} c_{\lambda, \delta}^{\prime} d_{\lambda}\left\|\widehat{f}_{\lambda}\right\|^{2} .
$$

Assuming $\mathbb{N S}_{\delta}(f) \leq \epsilon$, we have:

$$
\begin{aligned}
\epsilon \geq \frac{1}{2}-\frac{1}{2} \sum_{\lambda} c_{\lambda, \delta}^{\prime}\left\|\widehat{f}_{\lambda}\right\|^{2} & =\frac{1}{2}\left(\sum_{\lambda} d_{\lambda}\left\|\widehat{f}_{\lambda}\right\|^{2}-\sum_{\lambda} c_{\lambda, \delta}^{\prime} d_{\lambda}\left\|\widehat{f}_{\lambda}\right\|^{2}\right) \\
& =\frac{1}{2}\left(\sum_{\lambda}\left(1-c_{\lambda, \delta}^{\prime}\right) d_{\lambda}\left\|\widehat{f_{\lambda}}\right\|^{2}\right) \geq \frac{1}{2}\left(\sum_{\lambda: \lambda_{1} \leq n-1 / \delta}\left(1-c_{\lambda, \delta}^{\prime}\right) d_{\lambda}\left\|\widehat{f}_{\lambda}\right\|^{2}\right),
\end{aligned}
$$

where we used Parseval's identity in the first equality. 
We maximize $c_{\lambda, \delta}^{\prime}$ over $\lambda \vdash n$ where $\lambda_{1} \leq n-1 / \delta$. By Corollary 6.6, the maximum will occur at $\mu=(n-\lceil 1 / \delta\rceil,\lceil 1 / \delta\rceil)$.

By Corollary 6.8, we have $c_{\mu, \delta}^{\prime} \leq \mathbf{E}\left[\left(\frac{\operatorname{dim} \mu /(2)}{\operatorname{dim} \mu}\right)^{\boldsymbol{k}-1}\right]$. Using the moment generating function for the binomial distribution, we have

$$
c_{\mu, \delta}^{\prime} \leq \frac{\operatorname{dim} \mu}{\operatorname{dim} \mu /(2)}\left(1-\delta\left(1-\frac{\operatorname{dim} \mu /(2)}{\operatorname{dim} \mu}\right)\right)^{n} .
$$

We analyze the quantity $1-\frac{\operatorname{dim} \lambda /(2)}{\operatorname{dim} \lambda}$ using Equation (6), simple algebra, and the fact that $\lambda_{1}+\lambda_{2}=n$ :

$$
\begin{aligned}
1-\frac{\operatorname{dim} \lambda /(2)}{\operatorname{dim} \lambda} & =\frac{n(n-1)-\lambda_{1}\left(\lambda_{1}-1\right)-\lambda_{2}\left(\lambda_{1}-1\right)-\lambda_{2}\left(\lambda_{2}-1\right)}{n(n-1)} \\
& =\frac{n(n-1)-n\left(\lambda_{1}-1\right)-\lambda_{2}\left(\lambda_{2}-1\right)}{n(n-1)} \\
& =\frac{n \lambda_{2}-\lambda_{2}\left(\lambda_{2}-1\right)}{n(n-1)} \\
& =\frac{\left(\lambda_{2}\left(\lambda_{1}+1\right)\right.}{n(n-1)} .
\end{aligned}
$$

For $\mu=(n-\lceil 1 / \delta\rceil,\lceil 1 / \delta\rceil)$ we have $1-\frac{\operatorname{dim} \mu /(2)}{\operatorname{dim} \mu} \geq 0.9 /(\delta n)$. Further,

$$
1-\frac{\operatorname{dim} \mu /(2)}{\operatorname{dim} \mu}=\frac{\lceil 1 / \delta\rceil(n-\lceil 1 / \delta\rceil+1)}{n(n-1)} \leq \frac{((n+1) / 2)^{2}}{n(n-1)}=\frac{(n+1)^{2}}{4 n(n-1)} \leq 36 / 80,
$$

assuming $n \geq 5$. Thus $\frac{\operatorname{dim} \mu}{\operatorname{dim} \mu /(2)} \leq 80 / 44 \leq 2$, so $c_{\mu, \delta}^{\prime} \leq 2(1-\delta(0.9 /(\delta n)))^{n} \leq 2 e^{-0.9}$, which is strictly less than 1 . It follows that

$$
\epsilon \geq \frac{1}{2}\left(\sum_{\lambda: \lambda_{1} \leq n-1 / \delta}\left(1-c_{\lambda, \delta}^{\prime}\right) d_{\lambda}\left\|\widehat{f}_{\lambda}\right\|^{2}\right) \geq \frac{1}{2}\left(\sum_{\lambda: \lambda_{1} \leq n-1 / \delta}\left(1-2 e^{-0.9}\right) d_{\lambda}\left\|\widehat{f}_{\lambda}\right\|^{2}\right)
$$

and therefore

$$
\sum_{\lambda: \lambda_{1} \leq n-10 / \delta} d_{\lambda}\left\|\widehat{f}_{\lambda}\right\|^{2} \leq \sum_{\lambda: \lambda_{1} \leq n-1 / \delta} d_{\lambda}\left\|\widehat{f}_{\lambda}\right\|^{2} \leq \frac{2}{1-2 e^{-0.9}} \epsilon
$$

as claimed.

We remind the reader that we briefly discuss another generalization of noise sensitivity using random walks on Cayley graphs in Appendix A, and the model there has been studied more in the literature.

\section{APPLICATIONS TO LEARNING}

In this section, we prove our main theorems regarding agnostic learning.

Theorem 7.1. Let $\mathcal{C}$ be the class of linear threshold functions over $S_{n}$. There is an algorithm that agnostically learns with respect to $\mathcal{C}$ under the uniform distribution on $S_{n}$, using $n^{O\left(1 / \epsilon^{4}\right)}$ time and examples.

Proof. Combining Theorems 6.9 and 5.4 (Theorem 3.6), we get that $\mathcal{C}$ is $\epsilon^{2}$ concentrated up to degree $O\left(1 / \epsilon^{4}\right)$. It follows that the algorithm of Kalai et al. [2008] 
agnostically learns $\mathcal{C}$ under the uniform distribution on $S_{n}$ using $n^{O\left(1 / \epsilon^{4}\right)}$ time and examples.

TheOREM 7.2 (TheOREM 3.3). Let $\mathcal{C}$ be the class of functions of $k$ linear threshold functions over $S_{n}$. There is an algorithm that agnostically learns $\mathcal{C}$ under the uniform distribution on $\mathcal{D}$, using $n^{O\left(k^{2} / \epsilon^{4}\right)}$ time and examples.

Proof. Since $\mathbb{N S}_{\delta}(f)$ is a probability, by the union bound $\mathbb{N S}_{\delta}\left(h\left(f_{1}, f_{2}, \ldots, f_{k}\right)\right) \leq$ $\sum_{i=1}^{k} \mathbb{N S}_{\delta}\left(f_{i}\right)$. If each $f_{i}$ is a linear threshold function, then we set $\delta=\epsilon^{4} / k^{2}$ so $\mathbb{N S}_{\delta}\left(f_{i}\right) \leq$ $\epsilon^{2} / k$ for each $i$, so we have $\mathbb{N S}_{\delta}\left(h\left(f_{1}, f_{2}, \ldots, f_{k}\right)\right) \leq \epsilon^{2}$. The result follows similarly to the previous proof.

For a string $x \in\{0,1\}^{n}$ and bit $b \in\{0,1\}$, let $I_{b}(x)$ be the set $\left\{i: x_{i}=b\right\}$. Define $\mathcal{U}_{m}$ to be the uniform distribution over strings where $\left|I_{1}(x)\right|=m$.

THEOREM 7.3. There is an algorithm running in time $n^{O\left(1 / \epsilon^{4}\right)}$ for agnostically learning with respect to the class of halfspaces over $\{0,1\}^{m}$ under the distribution $\mathcal{U}_{m}$.

Proof. Let $f^{*}(x)=\operatorname{sgn}\left(\sum_{i} w_{i} x_{i}-\theta\right)$ be the most accurate linear threshold function in computing the target function $t$. From the previous section, we know $\mathbb{N S}_{\epsilon^{4}}\left(f^{*}(x)\right) \leq$ $O\left(\epsilon^{2}\right)$, so $f^{*}$ is $O\left(\epsilon^{2}\right)$-concentrated to degree $1 / \epsilon^{4}$.

Convert every example $\langle x, f(x)\rangle$ where $x$ is drawn from $\mathcal{U}_{m}$ to a permutation $\sigma$ by uniformly randomly assigning a random permutation from $I_{1}(x) \rightarrow[k]$ and $I_{0}(x) \rightarrow[n] \backslash[k]$. Note that there exists a linear threshold function over permutations that classifies at least as well as $f^{*}$, since the classifier

$$
g(\sigma)=\operatorname{sgn}\left(\sum_{i} w_{i} \mathbf{1}[\sigma(i) \in[k]]-\theta\right)
$$

is a linear threshold function and is consistent with $f^{*}$ over strings in $\mathcal{U}_{m}$. Further, every permutation is equally likely, so the resulting marginal distribution of the first component of the examples under the transformation is the uniform distribution over $S_{n}$. Therefore, the SVM algorithm of Kalai et al. [2008] described earlier will output a hypothesis with error at most $\epsilon$ worse than the error of $g$. To classify future instances, we can convert from a bit string to a permutation in the same way.

THEOREM 7.4. There is an algorithm running in time $n^{O\left(k^{2} / \epsilon^{4}\right)}$ for agnostically learning with respect to the class of functions of $k$ halfspaces over $\{0,1\}^{n}$ under the distribution $\mathcal{U}_{m}$.

Proof. The proof is nearly identical to the proof of Theorem 3.3/Theorem 7.2, relying on Theorem 7.3 instead of Theorem 7.1.

One way of viewing the above theorem is that the algorithm learns under the uniform distribution over all permutations of the string $1^{m} 0^{n-m}$. This reduction can be applied to learn over all permutations of any $n$-character string over any alphabet. The symmetric group case is the case where all the characters in the string are distinct; we take $X=[n]$, and the distribution is uniform over all permutations of $(1,2, \ldots, n)$. We can apply our theorems to learning over permutation-invariant distributions.

TheOREM 7.5 (TheORem 3.1). Let $\mathcal{D}$ be any permutation-invariant distribution over $\{0,1\}^{n}$. There is an algorithm running in time $n^{O\left(k^{2} / \epsilon^{4}\right)}$ for agnostically learning with respect to the class of functions of $k$ halfspaces over $\{0,1\}^{n}$ under the distribution $\mathcal{D}$.

Proof. We partition the examples into $n+1$ bins depending on the number of 1 's in the example. Thus, the problem is reduced to learning over $\mathcal{U}_{0}, \mathcal{U}_{1}, \ldots, \mathcal{U}_{n}$. We then run 
the learning algorithm for each bin once enough examples are seen. We note that we might have to account for some loss due to an insufficient number of examples in some bin, but this can be factored into the error parameter $\epsilon$; details can be found in Blais et al. [2010].

To extend this to distributions over $\{1,2, \ldots, B\}^{n}$, we first define linear threshold functions for this domain. This definition is consistent with the definition we made earlier.

Definition 7.6. We say that $f:\{1,2, \ldots, B\}^{n} \rightarrow\{-1,1\}$ is a linear threshold function if $f$ can be expressed as

$$
f(x)=\operatorname{sgn}\left(\sum_{i, j} w_{i j} \mathbf{1}\left[x_{i}=j\right]-\theta\right)
$$

for some scalar $\theta$ and scalars $w_{i j}$ with $1 \leq i \leq n$ and $1 \leq j \leq B$.

Theorem 7.7 (Theorem 3.5). Let $\mathcal{D}$ be any permutation-invariant distribution over $\{1,2, \ldots, B\}^{n}$ for constant $B$. There is an algorithm running in time $n^{O\left(k^{2} / \epsilon^{4}+B\right)}$ for agnostically learning with respect to the class of functions of $k$ linear threshold functions over $\{0,1\}^{n}$ under the distribution $\mathcal{D}$.

Proof. The proof is virtually the same as the proof of Theorem 3.1/Theorem 7.5, except that the number of bins is now $\left(\begin{array}{c}n+B-1 \\ B-1\end{array}\right)=\operatorname{poly}\left(n^{B}\right)$, via a "stars and bars" counting argument.

\section{APPENDIX}

\section{A. NOISE SENSITIVITY VIA RANDOM WALKS}

In this section, we outline what might be the "expected" definition of noise sensitivity in our setting. We start with some preliminaries.

Given a group $G$ and a set of generators $U$, the Cayley graph $\operatorname{Cay}(G, U)$ is the graph whose vertices are elements of $G$, and $g_{1}$ is adjacent to $g_{2}$ if and only if there is an element of $u \in U$ such that $g_{1}=u g_{2}$. We will consider random walks on these graphs.

For a function $f: G \rightarrow \mathbb{R}$, for each $u \in U$, we define $L_{u} f(g)=f(g)-f(g u)$, and the Laplacian $L=\frac{1}{|U|} \sum_{u \in U} L_{u}$. Then $L=\mathrm{id}-K$, where $K$ is the Markov operator or transition matrix for the random walk. We will denote the eigenvalues of $L$ as $0=\lambda_{0}<\lambda_{1} \leq \lambda_{2} \leq \cdots \lambda_{|G|-1}$, with corresponding eigenfunctions $1 \equiv \psi_{0}, \psi_{1}, \ldots, \psi_{|G|-1}$.

The continuous-time Markov semigroup operator $H_{t}$ is defined in the following way: For $g \in G$ and $f: G \rightarrow \mathbb{R}, H_{t} f(g)=\mathbf{E}_{\boldsymbol{h}}[f(\boldsymbol{h})]$, where $\boldsymbol{h}$ is generated from $g$ by taking a random walk of length $\alpha \sim \operatorname{Poisson}(t)$. The semigroup property is that $H_{s} H_{t}=$ $H_{s+t}$. An equivalent definition using the Laplacian is $H_{t}=e^{-t L}$. The functions $\psi_{i}$ are eigenfunctions of $H_{t}$ as well; the corresponding eigenvalue is $e^{-t \lambda_{i}}$.

We will first consider the hypercube. If we take $G=\mathbb{Z}_{2}^{n}$ and $U$ to be the set of unit vectors $e_{i}$ for $i \in[n]$, then $\operatorname{Cay}(G, U)$ is the $n$-dimensional hypercube. Each step of the random walk selects a random coordinate and flips it. We recall that the notion of rerandomizing a coordinate with probability $\delta$ is equivalent to flipping a coordinate with probability $\delta / 2$. For $0 \leq \delta<1$, let $\beta$ be the number of objects selected an odd number of times when selecting Poisson $(t)$ with replacement from a set of $n$ objects. Note that flipping a bit an odd number of times results in a flip, while an even number of flips does nothing. Coupon collector arguments yield that $\beta \sim \operatorname{Binomial}\left(n, \frac{1}{2}-\frac{1}{2} e^{-2 t / n}\right)$. We 
set $\frac{1}{2}-\frac{1}{2} e^{-2 t / n}=\delta / 2$, so $e^{-2 t} / n=1-\delta$. Then

$$
\left\langle f, H_{t} f\right\rangle=\sum_{\alpha \in \mathbb{Z}_{2}^{n}} e^{-2|\alpha| t / n} \hat{f}(\alpha)^{2}=\sum_{\alpha \in \mathbb{Z}_{2}^{n}}(1-\delta)^{|\alpha|} \hat{f}(\alpha)^{2}
$$

and thus, setting $t$ so $e^{-2 t / n}=1-\delta$, we have $\mathbb{N S}_{\delta}(f)=\frac{1}{2}-\frac{1}{2}\left\langle f, H_{t} f\right\rangle=\frac{1}{2}-\frac{1}{2} \sum_{\alpha}(1-$ $\delta)^{|\alpha|} \hat{f}(\alpha)^{2}$, which is the formula from Section 4.2.

This line of thought naturally yields a generalization to the case of the symmetric group. Let $U \subseteq S_{n}$ be the set of the $\left(\begin{array}{l}n \\ 2\end{array}\right)$ transpositions. Then $U$ is a generating set for $S_{n}$, and we consider the resulting Cayley graph $\operatorname{Cay}\left(S_{n}, U\right)$. Let $\boldsymbol{\alpha} \sim \operatorname{Poisson}(t)$, and we will reparametrize $t=\delta n$ for consistency with the main body of this article. Let $\bar{N}_{\delta}$ be the distribution whose samples are generated by a random walk of length Poisson $(\delta n)$ starting at the identity permutation. Replacing $N_{\delta}$ with $\bar{N}_{\delta}$ in the previous sections is straightforward; the distribution $\bar{N}_{\delta}$ is a class distribution, so much of the setup in Section 6 applies and most of the technical work in Section 6 can be avoided.

The matrix entries of a complete set of irreducible representations are eigenfunctions of the Laplacian. From Diaconis [1988], the eigenvalue for such a function coming from a representation corresponding to the partition $\lambda \vdash n$ is $2-2 \frac{\operatorname{dim} \lambda /(2)}{\operatorname{dim} \lambda}$. It follows that the eigenvalues of $H_{t} f$ are $\exp \left(-t\left(2-2 \frac{\operatorname{dim} \lambda /(2)}{\operatorname{dim} \lambda}\right)\right)$.

For consistency with the main body, we will reparametrize to use a noise parameter $0 \leq \delta<1$, such that $e^{-2 t / n}=1-\delta$. Under this setting, $H_{t}$ plays the role of $T_{\delta}$ from the main narrative, and we have

$$
\bar{c}_{\lambda, \delta}^{\prime}=(1-\delta)^{r} \text { where } r=\left(1-\frac{\operatorname{dim} \lambda /(2)}{\operatorname{dim} \lambda}\right) n,
$$

where $\bar{c}_{\lambda, \delta}^{\prime}$ is the natural generalization of $c_{\lambda, \delta}^{\prime}$ to the alternate noise operator $\bar{N}_{\delta}$. Note that Inequality (8) looks quite similar to this equation. With this expression for $\bar{c}_{\lambda, \delta}^{\prime}$, the analogue of the proof of Theorem 6.9 proceeds in almost exactly the same manner.

We chose the presentation in the main body for two reasons: First, the combinatorial type of noise described in the main body appears to be new and hopefully will be useful in other settings. Second, we were unable to prove an analogue of Theorem 5.4 (regarding bounding noise sensitivity of linear threshold functions) without applying the results presented in Section 6.

\section{REFERENCES}

Eric Blais, Ryan O’Donnell, and Karl Wimmer. 2010. Polynomial regression under arbitrary product distributions. Mach. Learn. 80, 2 (2010), 273-294. http://www.springerlink.com/index/988211hq3v2q5171.pdf.

Dan Boneh. 1995. Learning using group representations. In Proceedings of the Eighth Annual Conference on Computational Learning Theory. ACM, 418-426. http://dl.acm.org/citation.cfm?id=225349

Persi Diaconis. 1988. Group representations in probability and statistics. Lecture Notes-Monograph Series 11 (1988), i-192. http://www.jstor.org/stable/10.2307/4355560.

David Ellis, Ehud Friedgut, and Haran Pilpel. 2011. Intersecting families of permutations. J. Am. Math. Soc. 24, 3 (2011), 649-682.

Vitaly Feldman and Pravesh Kothari. 2015. Agnostic learning of disjunctions on symmetric distributions. $J$. Mach. Learn. Res. 16 (2015), 3455-3467.

William Fulton. 1997. Young Tableaux: With Applications to Representation Theory and Geometry. Vol. 35. Cambridge University Press.

Jonathan Huang, Carlos Guestrin, and Leonidas Guibas. 2009. Fourier theoretic probabilistic inference over permutations. J. Mach. Learn. Res. 10 (2009), 997-1070. http://dl.acm.org/citation.cfm?id=1577106

G. D. James and Adalbert Kerber. 1981. The Representation Theory of the Symmetric Group. Addison-Wesley, Reading, MA. pp. xxviii, 510. 
Adam Tauman Kalai, Adam R. Klivans, Yishay Mansour, and Rocco A. Servedio. 2008. Agnostically learning halfspaces. SIAM J. Comput. 37, 6 (2008), 1777-1805. http://epubs.siam.org/doi/abs/10.1137/060649057.

Adam Tauman Kalai, Alex Samorodnitsky, and Shang-Hua Teng. 2009. Learning and smoothed analysis. In Foundations of Computer Science, 2009. FOCS'09. 50th Annual IEEE Symposium on. IEEE, 395-404. http://ieeexplore.ieee.org/xpls/abs_all.jsp?arnumber $=5438615$.

Daniel M. Kane, Adam Klivans, and Raghu Meka. 2013. Learning halfspaces under log-concave densities: Polynomial approximations and moment matching. In COLT. 522-545.

Jovan Karamata. 1932. Sur une inégalité rélative aux fonctions convexes. Publ. Math. Univ. Belgrade 1 (1932), 145-148.

Michael J. Kearns, Robert E. Schapire, and Linda M. Sellie. 1994. Toward efficient agnostic learning. Mach. Learn. 17, 2-3 (1994), 115-141. http://link.springer.com/article/10.1007/BF00993468.

Adam R. Klivans, Ryan O'Donnell, and Rocco A. Servedio. 2004. Learning intersections and thresholds of halfspaces. J. Comput. Syst. Sci. 68, 4 (2004), 808-840.

Risi Kondor and Karsten M. Borgwardt. 2008. The skew spectrum of graphs. In Proceedings of the 25th International Conference on Machine Learning. ACM, 496-503. http://dl.acm.org/citation.cfm?id=1390219

Risi Kondor, Andrew Howard, and Tony Jebara. 2007. Multi-object tracking with representations of the symmetric group. In International Conference on Artificial Intelligence and Statistics. 211-218.

Nathan Linial, Yishay Mansour, and Noam Nisan. 1993. Constant depth circuits, Fourier transform, and learnability. J. ACM 40, 3 (1993), 607-620. http://dl.acm.org/citation.cfm?id=174138

Ryan O'Donnell. 2007. Lecture notes on analysis of boolean functions. (2007).

Ryan O'Donnell and Karl Wimmer. 2009. KKL, Kruskal-Katona, and monotone nets. In Foundations of Computer Science, 2009. FOCS'09. 50th Annual IEEE Symposium on. IEEE, 725-734. http://ieeexplore. ieee.org/xpls/abs_all.jsp?arnumber $=5438582$.

Andrei Okounkov and Grigori Olshanski. 1998. Shifted Schur functions. St. Petersburg Math. J. 9, 2 (1998), 239-300.

Yuval Peres. 2004. Noise stability of weighted majority. arXiv preprint math / 0412377 (2004). http://arxiv.org/ $\mathrm{abs} / \mathrm{math} / 0412377$.

Pannagadatta K. Shivaswamy and Tony Jebara. 2006. Permutation invariant SVMs. In Proceedings of the 23rd International Conference on Machine Learning. ACM, 817-824. http://dl.acm.org/citation. $\mathrm{cfm} ? \mathrm{id}=1143947$

Received January 2015; accepted November 2015 